\title{
On the Characterization and Selection of Diverse Conformational Ensembles, with Applications to Flexible Docking
}

\author{
Sébastien Loriot and Sushant Sachdeva and Karine Bastard and Chantal Prévost and Frédéric Cazals
}

\begin{abstract}
To address challenging flexible docking problems, a number of docking algorithms pre-generate large collections of candidate conformers. To further remove the redundancy from such ensembles, a central question in this context is the following one: report a selection of conformers maximizing some geometric diversity criterion. In this context, we make three contributions.

First, we tackle this problem resorting to geometric optimization so as to report selections maximizing the molecular volume or molecular surface area (MSA) of the selection. Greedy strategies are developed, together with approximation bounds.

Second, to assess the efficacy of our algorithms, we investigate two conformer ensembles corresponding to a flexible loop of four protein complexes. By focusing on the MSA of the selection, we show that our strategy matches the MSA of standard selection methods, but resorting to a number of conformers between one and two orders of magnitude smaller. This observation is qualitatively explained using the Betti numbers of the union of balls of the selection.

Finally, we replace the conformer selection problem in the context of multiple-copy flexible docking. On the systems above, we show that using the loops selected by our strategy can significantly improve the result of the docking process.
\end{abstract}

Index Terms-Flexibility, conformer selection, flexible docking, geometric optimization, Van der Waals models.

Abbreviations: MSA: molecular surface area, VdW: Van der Waals.

\section{INTRODUCTION}

\subsection{On the Importance of Diverse Conformational Ensembles}

Ensembles in molecular modeling. Protein-protein interactions are paramount to all biological processes, but their prediction from unbound geometries faces major difficulties, as evidenced in the CAPRI experiment, by the low number of medium and high predictions carried out on flexible systems-as opposed to incorrect and acceptable ones [1]. Since proteins are intrinsically flexible,

K. Bastard is with Biotechnologie-Biocatalyse-Biorégulation, Université de Nantes - CNRS, France.

F. Cazals is with INRIA Sophia-Antipolis, Algorithms-Biology-Structure, France.

S. Loriot is with IMB - Université de Bourgogne, France and INRIA SophiaAntipolis, Algorithms-Biology-Structure, France.

C. Prévot is with Institut de Biologie Physico-Chimique, Paris, France.

S. Sachdeva is with IIT Bombay, India.

Corresponding author: Frederic.Cazals@inria.fr. they continuously undergo conformational changes over time, or in an equivalent way, they exist at a given time as an ensemble of conformations in equilibrium. During their exploration of the conformational space, they preferably occupy regions which are characterized by low free energies. For proteins of moderate size undergoing small amplitude movements occurring in time scales of tens of nanoseconds, conformational changes can be investigated using molecular dynamics, namely by numerically integrating Newton's equations of motion. For more complex cases, where flexibility applies to large parts of the protein backbone or where the amplitude of the movement is important, discrete ensembles of conformations known as conformers can be pre-generated and considered simultaneously. This representation is particularly appropriate when dealing with macromolecular docking. In the case of association, one indeed wishes to predict the best possible bound geometry of two flexible objects, which subsumes exploring the relative position and orientation of the partners, but also their conformational space so as to pack the interface. In the Monod-Wyman-Changeux interpretation [2], the unbound proteins are considered as two collections of conformers in thermodynamic equilibrium. When the partners bind, the equilibrium is shifted toward the structure observed in the complex. Implementing this strategy may be done at the global (i.e. protein) scale [3], local (i.e. side chain) scale [4], or intermediate (i.e. loops or domains) scale [5].

Generating and selecting conformers: energy versus geometry. Representing flexibility through an ensemble of conformers is computationally feasible only if this number is not too important. It is therefore essential for this reduced number of conformers to be as representative as possible of the conformational space available to the flexible molecule or molecular fragment. More generally, conformers being of interest for a number of applications, which criteria (geometric or energetic) should one use to generate and/or select them? In a statistical viewpoint, energy should be the criteria of choice for generating ensembles representative of the thermodynamic equilibrium between conformations. However, 
this criteria is generally not tractable for several reasons.

First and foremost, the exhaustive exploration of the conformational space of large systems or of systems with large amplitude deformations is not possible. To keep calculations tractable, methods undertaking this task favor geometric calculations, and defer energy calculations to later stages [6], [7]. Second, when conformers are used to model a region of a protein, the energy associated to each conformer varies with its environment. In the case of docking for example, the energy of each copy depends upon its interactions with the partner of association (direct electrostatic or Van der Waals interactions, modification of the dielectric environment, desolvation energy). Therefore, weighting a conformer as if it were alone does not, in general, precisely account for its probability of occurrence. Third, it may happen that the energy landscape associated to a flexible protein is rather flat, with very small energy barriers between the conformers. In contrast to flipping between well separated conformers, the protein flexible fragment can largely explore the available space. In this case, it is important to be able to sample exhaustively the space available to the flexible element.

In passing, we may also notice that the generation of diverse ensembles is a strategy of choice to simulate complex processes. For example, diverse ensembles generated using a repulsive umbrella potential have recently been used to investigate domain swapping [8].

\subsection{Contributions and Paper Overview}

Conformers: atomic and coarse models. Consider a collection $\mathcal{C}=\left\{C_{1}, \ldots, C_{n}\right\}$ of $n$ conformers (rotamers, protein loops, whole protein), each represented by a collection of balls, each ball being bounded by a sphere. This model is rather general, as the balls in Van der Waals (VdW) model may represent atoms, or may model residues. In this study, we shall use atomic and coarse protein models.

Problem addressed. As just argued, sampling the conformational space available is an important requirement. We actually wish to solve the following problem:

Given a pre-computed collection of $n$ conformers and an integer $s<n$, report a selection of $s$ conformers maximizing some geometric diversity criterion.

To specify the type of geometric criterion we have in mind, observe that the union of the balls of the conformers in the selection defines a volume, whose partition by the spheres bounding the balls is called a volumetric arrangement (also called volumetric decomposition). Similarly, the decomposition of each sphere by the intersection circles with other spheres defines a surface arrangement (also called surface decomposition). See Figs. 1 and 2 for a $2 D$ illustration. Using these arrangements, we investigate several geometric optimization problems whose output is the selection. These problems aim at maximizing the spatial occupancy of the selection, in

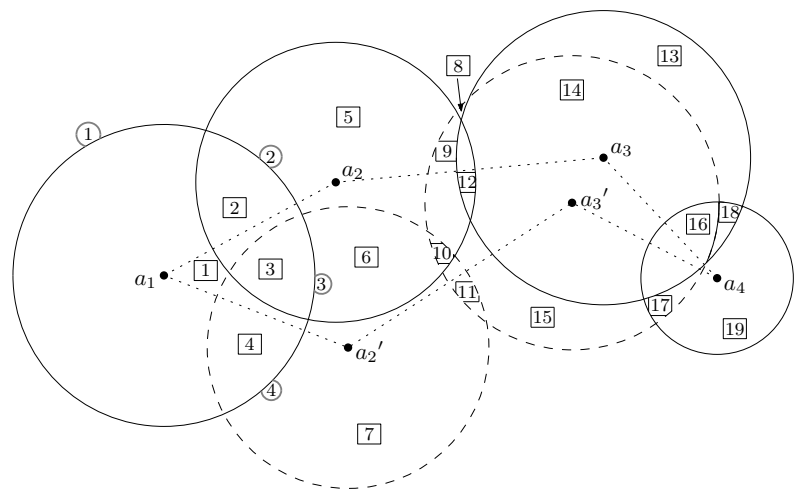

Fig. 1. Example of $2 D$ conformers, each consisting of four balls-first and fourth balls are common. The (two dimensional) volume occupied by the two conformers is decomposed into 19 cells (boxed numerals). The circled numerals feature the surface arrangement of the ball centered at $a_{1}$, based on intersections with neighboring balls.

several guises. For example, we may wish to report the $s$ conformers maximizing (i) the volume occupied by these conformers (ii) the molecular surface area (MSA) of the union of the conformers (see Fig. 4).

As an illustration, consider Fig. 3(a), which features 40 conformers of the flexible loop of a complex. A number of these loops are obviously redundant, and one would like to trim this set to select a diverse subset. Such a selection, generated by our algorithm, is presented on Fig. 3(b) and Fig. 4.

Paper overview. Two conformer selection problems phrased as geometric optimization problems are presented in section 2, together with a general strategy to solve them, the greedy strategy. In section 3, we focus on one such problem, namely that of reporting a selection maximizing the MSA area of the union of the conformers, and present the protein-protein complex used for the validation. A geometric and topological assessment of the diversity is presented in section 4, while an assessment of the quality of the conformers selected for flexible protein docking is presented in section 5. These assessments are conducted by comparing our algorithm, Greedy, to a contender named HClust based on a hierarchical clustering strategy. Upon concluding in section 6 , we provide the proofs of the theorems presented in the main text in appendices A and B, and further discuss the conformer generation methods used in appendix $\mathrm{C}$.

\section{Selecting Conformers: the Combina- TORIAL VIEWPOINT}

\subsection{Arrangements of Balls and Spheres: Volume and Surface Decompositions}

The spheres bounding the balls of a collection of conformers induce two decompositions: a decomposition of the volume occupied by the balls; and a decomposition of each sphere into spherical patches. More precisely, 

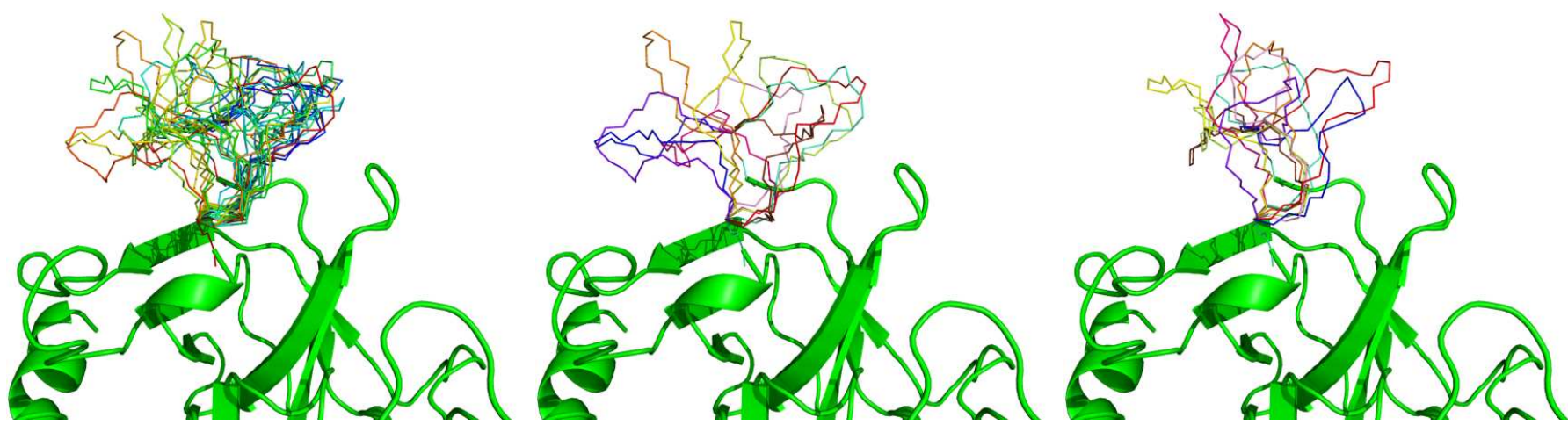

Fig. 3. Selecting diverse conformational ensembles from a pool of conformers of a flexible loop-PDB code 1BTH. From left to right: (a) backbone of the receptor in cartoon mode, with 40 conformers from the pool (b) backbone together with 10 conformers selected by our algorithm—called Greedy (c) backbone with 10 conformers selected by a standard hierarchical clustering algorithm—called HClust.

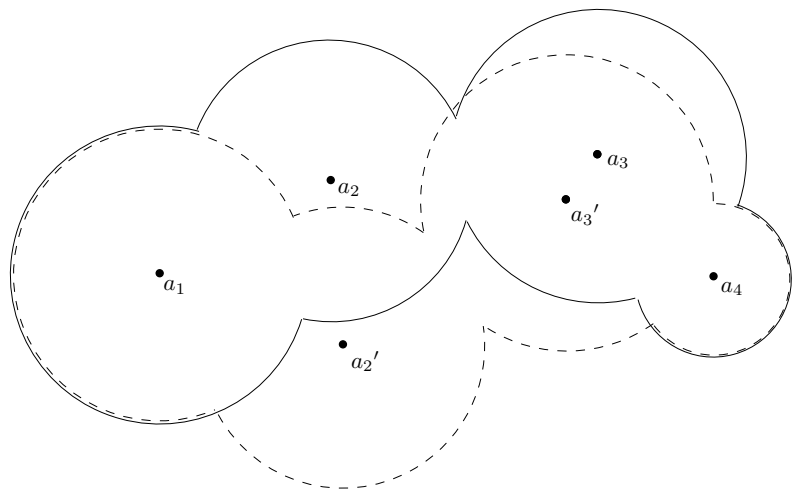

Fig. 2. The boundary of the union of balls of the two conformers of Fig. 1, respectively in solid and dashed lines.
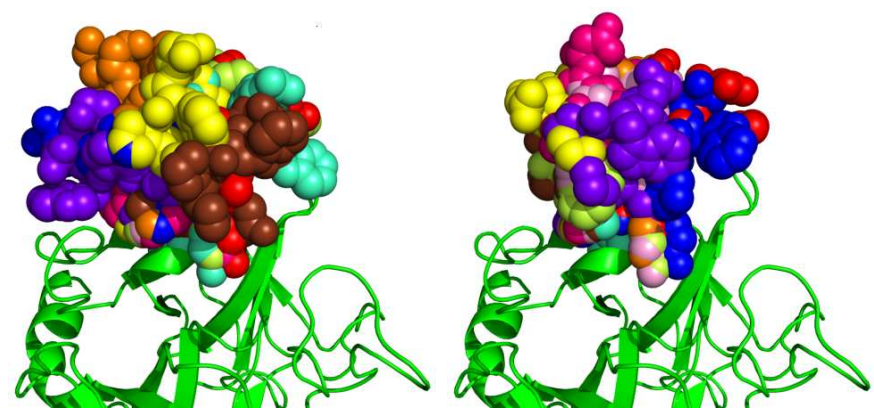

Fig. 4. Follow-up to Fig. 3: Van der Waals representations of the 10 conformers presented on Fig. 3(b) and Fig. 3 (c). Notice that conformers of the former set are well separated, while those of the latter are cluttered.

consider the three-dimensional domain spanned by the conformers, that is the union of their defining balls. The decomposition of this volume induced by the spheres is called a volumetric arrangement (or volumetric decomposition). This arrangement consists of a collection of cells $\mathcal{A}=\left\{A_{i}\right\}$, such that the interior of each cell is connected. Each such cell is bounded by $2 D$ cells called surface patches, found on the spheres bounding the balls. On a given sphere, these patches are induced by the intersection circles with neighboring spheres. The collection $\mathcal{P}=\left\{P_{i}\right\}$ of all such patches defines a surface arrangement (or surface decomposition). See Fig. 1 for an illustration.

\subsection{Optimization Problems}

Problems statements. We shall be concerned with two classes of combinatorial optimization problems arising from geometric representations of molecular shapes. To state these problems from the combinatorial viewpoint (see section 2.3 for the connexion with conformers), assume we are given a base set $\mathcal{U}=\left\{U_{i}\right\}_{i=1, \ldots, m}$ consisting of cells (think cells of the volume or surface arrangement), and a collection of sets $\mathcal{C}=\left\{C_{i}\right\}_{i=1, \ldots, n}$ called the pool (think conformers). For a subset $\mathcal{S} \subset \mathcal{C}$, denote $\cup_{\mathcal{S}} C_{j}$ the union of the sets in $\mathcal{S}$. Cells and sets shall be subsets of $\mathbb{R}^{3}$, so that the inclusion of a cell $U_{i}$ in a set $C_{j}$ is naturally defined.

For the first class of problems, assume we are given a weight function $w$, i.e. a real valued function defined over the cells. Denote $\left(\begin{array}{l}\mathcal{C} \\ s\end{array}\right)$ the set of all subsets of $\mathcal{C}$ of size $s$. We define:

Problem 1. Given a weight function $w$, find a subset $\hat{\mathcal{S}}$ of $\mathcal{C}$ of size s, called the selection, such that:

$$
\hat{\mathcal{S}}=\arg \max _{\mathcal{S} \in\left(\begin{array}{c}
c \\
s
\end{array}\right)} w(\mathcal{S}) \text {, with } w(\mathcal{S})=\sum_{U_{i} \in \cup_{\mathcal{S}} C_{j}} w\left(U_{i}\right) .
$$

For the second class of problems, assume the weight function depends not only on the cells of the decomposition, but also in the selection $\mathcal{S}$, which we denote $w_{\mathcal{S}}\left(U_{i}\right)$. We wish to solve:

Problem 2. Given a weight function $w_{\mathcal{S}}$, find a subset $\hat{\mathcal{S}}$ of $\mathcal{C}$ of size s, called the selection, such that:

$$
\hat{\mathcal{S}}=\arg \max _{\mathcal{S} \in\left(\begin{array}{l}
\mathcal{C} \\
s
\end{array}\right)} w(\mathcal{S}) \text {, with } w(\mathcal{S})=\sum_{U_{i} \in \cup_{\mathcal{S}} C_{j}} w_{\mathcal{S}}\left(U_{i}\right) .
$$

Complexity issues. Our problems are intimately related to max- $k$ cover. Given a set $\mathcal{U}$ of $n$ points, and a collection 
$\mathcal{C}$ of subsets of $\mathcal{U}$, max- $k$ cover is the problem of selecting $k$ subsets from $\mathcal{C}$ such that their union contains as many points from $\mathcal{U}$ as possible [9], [10]. (There is some confusion in the literature, as this problem is called set cover in [11]. In fact, the partial set cover problem consists of picking the minimum number of sets in $\mathcal{C}$ so as to contain at least $k$ elements from $\mathcal{U}$.) If the weight function $w$ assigns a unit weight to all cells, then Problem 1 reduces to max- $k$ cover. Since this is a NP-Complete problem, we cannot expect to have an exact algorithm for our problem that works in time polynomial in both $|\mathcal{C}|$ and $s$.

On the other hand, for a fixed $s$, the search space of all possible combinations of conformers is $\mathcal{O}\left(|\mathcal{C}|^{s}\right)$. Hence, for a fixed $s$ the problem is in $\mathbf{P}$. However, even for a modest $s$, the brute force method is too costly to be used in practice. Section 2.4 presents an approximate strategy whose time complexity does not grow exponentially with $s$.

\subsection{Instantiations to Conformer Selection}

Problem 1 from volumetric decomposition. Consider the base set $\mathcal{A}$ whose cells are those of the $3 D$ arrangement. In Eq. (1), let $w$ be some general function defined on the cells of the volumetric decomposition, for example the standard Euclidean volume. For conformer selection, optimizing the volume of a selection is a direct way to ascertain a good spatial diversity, since overlaps between conformers are minimized.

Problem 2 from surface decomposition. Consider the base set $\mathcal{P}=\left\{P_{i}\right\}$ whose cells are those of the $2 D$ arrangements. Special cells of this arrangement are those which are exposed, i.e. contribute to the boundary of the union of balls. Focusing on these patches yields an instantiation of Problem 2, the dependence upon the selection $\mathcal{S}$ consisting of discarding the patches which are not exposed with respect to the selection. That is, in Eq. (2), $w_{\mathcal{S}}\left(P_{i}\right)$ stands for some general function defined on the surface patches found on the boundary of the union of balls. For example $w_{\mathcal{S}}\left(P_{i}\right)=$ surface area of patch $P_{i}$ iff $P_{i}$ is found on the boundary of the union, and 0 otherwise.

Practically, we shall be dealing with atomic and coarse models. By molecular surface, we refer to the Van der Waals surface for the former, and to the boundary of the union for the latter-coarse models are specified in section 3.1.

Interestingly, maximizing the boundary surface of the selection is an indirect way to ascertain some diversity, since the overlap between conformers is minimized. Notice, though, that as opposed to the volume, the boundary surface area is not a monotonic function of the number of conformers. That is, for two selections $S_{1}$ and $S_{2}$ with $S_{1} \subset S_{2}$, one has $\operatorname{volume}\left(S_{2}\right) \geq \operatorname{volume}\left(S_{1}\right)$, a property that may not hold for the boundary surface area.

\subsection{The Greedy Strategy}

\subsubsection{The Strategy and its Guarantees}

To solve our optimization problems, an obvious approach is the greedy method. The greedy algorithm performs $s$ steps, selecting at each step an element $C_{j}$ of $\mathcal{C}$, that has not yet been selected, and that maximizes the sum of the weights of the cells being added. In other words, at each step, the algorithm selects a $C_{j}$ that maximizes the weight of the union of the $C_{j}$.

Unfortunately, the selection obtained this way may not realize the optimum solution. As an example consider Fig. 5: For selecting two conformers, the optimum choice has a weight of 14 whereas the greedy method gives us a collection with a weight of 12 . To scale this performance, one resorts to the approximation ratio, that is the ratio between the solution returned and the optimal one. For max- $k$ cover, this ratio is known to be of $1-1 / e$, and is actually tight [12], [13], [11], [10].
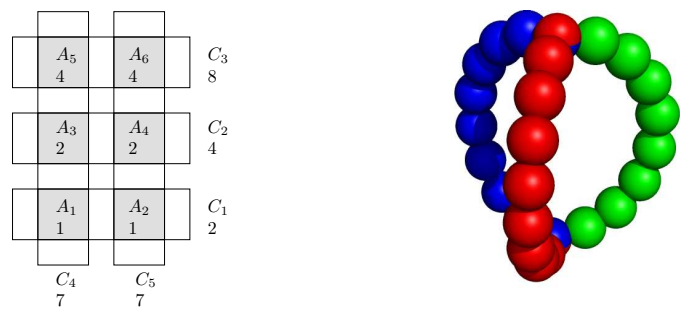

Fig. 5. Selecting two con- Fig. 6. Three fictitious conformers out of $C_{1}, \ldots, C_{5}$ formers of a protein loop: by the greedy strategy fails The surface of the union to report the optimal solu- of the three conformers detion. The shaded regions fines a double torus, i.e. have the weights as indi- a surface of genus two. cated and the unshaded Compare to Fig. 3(b). regions have null weights.

\subsubsection{Application to Conformer Selections}

Volumetric decomposition, general weight $w$. Consider a volumetric decomposition as specified in section 2.1. The weighting scheme is called non-negative provided all weights are $\geq 0$. The approximation ratio of the greedy strategy and its optimality are usually proved in the uniform weight case [12], [13], [11], [10]. The following theorems, proved in appendix A, provide generalizations to non-negative weights:

Theorem 2.1. Consider a volumetric decomposition with non negative weights. For Problem 1, the greedy approach has an approximation guarantee of $1-(1-1 / s)^{s}>1-1 / e$.

Theorem 2.2. The greedy approach cannot perform better than $1-(1-1 / s)^{s}$.

Surface decomposition, boundary surface weight $w_{\mathcal{S}}$. For volumetric decompositions, the previous bound indicates one is always above $63 \%(1-1 / e)$ from the optimum. Unfortunately, as shown in appendix B.1, such a result does not hold for problem 2 : 
Observation 1. Consider a surface decomposition. For Problem 2, the greedy approach may have an approximation guarantee as bad as $1 / s^{2}$.

Practical considerations. In the following, we shall focus on the problem of optimizing the surface area rather then the volume of a selection for two reasons. First, we are not aware of any robust implementation to report the volume of a union of balls. A contrario, robust and optimized algorithms exist to handle surface arrangements [14], [15], [16]

\section{Material and Methods}

In this section, we introduce the models, concepts and algorithms used to make a geometric and topological assessment (section 4) and a docking assessment (section 5) of the selections.

\subsection{Data Sets and Conformer Generation Methods}

Protein models and their comparison. In addition to atomic protein models, we shall be dealing with coarse models. Following [17], given an atomic model, a coarse - residue-based model is obtained by replacing the sidechain by one or two pseudo-atoms, depending on the amino-acid type-the location of the $C_{\alpha}$ carbon does not change. To distinguish two models of a complex, say $1 \mathrm{BTH}$, we shall use the notations 1BTH-atomic (respectively 1BTH-coarse) for the all atom (respectively coarse) model.

A classical statistic used for comparing two proteins is the $C_{\alpha}$-rmsd, that is the standard deviation of the distance between the atomic positions of the $C_{\alpha}$ carbons of the two proteins. (Below, we shall use the $C_{\alpha}$-rmsd to specify algorithm HClust, and to compare the selection of Greedy and HClust.) While the $C_{\alpha}$-rmsd is a good measure to compare two (portions of) proteins, a finer statistic is called for to evaluate the interface of a putative complex proposed by a rigid docking algorithm. To meet this need, we shall use the interface rmsd, denoted Irmsd. To define it, call the two partners of the complex the ligand and the receptor, and assume that the receptor of the co-crystallized complex has been aligned with that of the putative one. The I-rmsd is the $C_{\alpha}$-rmsd restricted to selected atoms of the ligand: those identified in the native complex within a distance threshold of $7 \AA$ from the receptor [18], [5].

Protein loops. We study four flexible protein loops belonging to the protein-protein interface of four complexes, 1OAZ, 1CGI, 1BTH and 3HHR. For each complex, both the unbound and the bound i.e. co-crystallized structures of the partners are known, and the conformation of the studied loops differs between these two forms. Three of the complexes (1CGI, 1BTH and 3HHR) come from the non-redundant protein-protein docking benchmark [19]. Complexes 1BTH and 3HHR have been identified as difficult cases since no acceptable structure could be predicted in rigid body docking studies [19].
Complex 1OAZ has been added because of the known flexibility of its interface [20].

The four flexible loops differ by size and degree of variation between the bound and unbound forms, as characterized by the $C_{\alpha}$-rmsd between the bound and unbound forms. In complex $1 \mathrm{BTH}$, the 10 amino acid (aa) loop of the thrombin mutant bound to the pancreatic trypsin inhibitor undergoes a $5.7 \AA$ deviation; in complex 1CGI, the structure of the 11 aa loop of $\alpha$-Chymo-trypsinogen bound to pancreatic secretory trypsin inhibitor has not been resolved in the unbound form, showing a high degree of flexibility; in 1OAZ, the 12 aa loop of the Ige Fv Spe7 protein complexed with a recombinant thioredoxin only undergoes a $2.1 \AA$ deviation, while in $3 \mathrm{HHR}$, the 26 aa loop of the human growth hormone bound to the extracellular domain of its receptor presents a deviations of $5.5 \AA$.

Conformer generations methods. A number of algorithms exist to generate atomic loop geometries [21], [22], [7], [23], [24]. We selected Direx [23] and Loopy [21], which respectively generate dense and sparse (exploring more space) ensembles of conformers. (For completeness, an overview of the strategies used herein is presented in appendix C.1.)

To scale the diversity of the loop ensembles generated, we computed the MSA of the union of a collection of $n=500$ conformers for the four models. (The residues involved in the MSA calculation are those from the loops together with the two residues bounding the loop, which are shared by all conformers.) For atomic and coarse models, the ratio $M S A($ Loopy $) / M S A($ Direx $)$ spans the range $[1.79,4.16]$ and $[1.86,4.60]$ respectively, which clearly shows that the Loopy data set is less redundant and explores more space. See Table 2 in appendix for a full report.

Geometry versus energy. Conformer generation methods, when applied to flexible loops, disregard the geometry of the scaffold accommodating the loop. To avoid steric clashes within the loop and in-between the loop and its scaffold, having computed the potential energy of the system loop+scaffold thanks to a 100-steps energy minimization with GROMACS [25], we discarded two types of conformers. First, those featuring a large short range Lennard-Jones term, which witnesses steric clashes between the loop and the scaffold. Second, those featuring a large bonded energy - featuring clashes within the loop.

\subsection{Greedy Selection: Implementation}

The naive and priority-based versions. Denote $I_{i}$ the selection of $i$ conformers after $i$ steps of the greedy strategy, and let $R_{i}$ stand for the remaining candidates. Following Eq. (2), the naive way of computing $I_{i}$ consists of incrementally linearly scanning all possible solutions, that is

$$
I_{i}=\arg \max _{C_{j} \in R_{i-1}} w\left(I_{i-1} \cup\left\{C_{j}\right\}\right) .
$$


As proved in appendix B, the following complexity is worst-case optimal:

Theorem 3.1. The naive version of Algorithm Greedy has complexity $O\left(n s^{3}\right)$.

A more elaborate strategy consists of maintaining the increments associated to all candidates, so as to select the best one from a priority queue. To do so, one needs in particular the surface arrangements on all spheres, together with the inclusion information of spherical patches into the other conformers. To account for this information, which encodes the complexity of the surface arrangement, denoting $\mathbf{1}_{X}$ the characteristic function of the Boolean variable $X$, define

$$
\tau=\sum_{C_{i} \in \mathcal{C}} \sum_{S_{j} \in C_{i}} \sum_{P_{k}} \mathbf{1}_{S_{j}} \text { covers patch } P_{k} \text { or } P_{k} \text { lies on } S_{j},
$$

where $\mathcal{C}$ is the set of all conformers, $S_{j}$ a sphere of a conformer $C_{j}$ and $P_{k}$ a patch on a sphere of a conformer in $\mathcal{C}$.

This variant, presented in appendix B, satisfies:

Theorem 3.2. The priority-based version of Algorithm Greedy has amortized complexity $O(\tau+s \log n)$.

Implementations. The naive implementation was carried out using the Delaunay_3 and Alpha_shape_3 packages of the Computational Geometry Algorithms Library [26]. For the priority based version, we used the surface arrangements package described in [14], [15], [16], which is the only one, to the best of out knowledge, able to compute effectively the exact arrangement of circles on a sphere. In both cases, robustness issues are critical due to the density of conformers manipulated.

\subsection{Conformer Selection Methods}

We compare algorithm Greedy against one contender, Algorithm HClust, which is a hierarchical agglomerative clustering [27] method based on the average linkage, used for protein-protein docking [5]. (We also tested the single linkage and complete linkage strategies, which performed equally w.r.t. the MSA - data not shown.) Given a dissimilarity measure between two clusters (i.e. groups of conformers), the algorithm generates a binary tree encoding a sequence of nested partitions of the $n$ conformers. Notice the coarser (respectively the finer) partition features one cluster (respectively $n$ clusters) containing the $n$ conformers (respectively a single conformer). As dissimilarity, we use the $C_{\alpha}$-rmsd between pairs of conformers. Cutting this binary tree at an appropriate level provides the number of desired conformers, since we select one representative within each cluster. The representative selection was carried out through a two-stage process, namely (i) a fictitious average loop is computed: for $k$ conformers each consisting of $p$ balls centered at $c_{i, j}$, with $i=1, \ldots, k$ and $j=1, \ldots, p$, the fictitious loop consists of $p$ balls centered at $\overline{c_{j}}=\left(\sum_{i=1, \ldots, k} c_{i, j}\right) / k$; (ii) the representative is taken as the conformer from the cluster having the least $C_{\alpha^{-}}$rmsd with this fictitious loop.

\section{Diverse Ensembles: Geometric and TOPOLOGICAL ASSESSMENT}

In this section, we discuss geometric and topological quantities to characterize the diversity of an ensemble, and compare those produced by the Greedy and HClust algorithms on four protein models.

\subsection{Statistics of Interest: Geometry vs. Topology}

Comparing MSA. We first report on the MSA. To see how, for a given selection method $M$ (G: greedy; H: hierarchical), let $\mathcal{N}_{M}=\left\{I_{1}, \ldots, I_{n}\right\}$ be a collection of selections of increasing size, i.e. selection $I_{i}$ contains $i$ conformers. The greedy strategy provides a nested collection of selections, since the selection $I_{i+1}$ of size $i+1$ is the selection $I_{i}$ of size $i$ to which an additional conformer has been prepended. The nestedness does not hold for algorithm HClust, though. As explained in section 3.3, one indeed gains one conformer by splitting one cluster $K$ (corresponding to a node $n_{K}$ in the binary tree) into two clusters $K_{1}$ and $K_{2}$ (the sons of node $n_{K}$ in the binary tree). But the representative conformer $C_{i}$ of cluster $K$ may not be that of the cluster $\left(K_{1}\right.$ or $\left.K_{2}\right)$ the conformer $C_{i}$ belongs to.

To compare two collections of selections, both for the atomic and the coarse models, we report two sets of values. To see which, let $R_{M}$ be the maximum of the MSA obtained over all selections in $\mathcal{N}_{M}$, that is $R_{M}=\max _{I_{i} \in \mathcal{N}_{M}} M S A\left(I_{i}\right)$. First, we focus on the maxima of MSA reached, that is on the ratio $R_{G} / R_{H}$. Second, denote $n_{H_{x}}$ the smallest number of conformers required by algorithm $\mathrm{H}$ to get a MSA (say $A$ ) equal to $x \%$ of its maximum. Then, denote $n_{G}$ the least number of conformers required by the greedy strategy to get a MSA greater or equal to $A$. We report $n_{H_{x}} / n_{G}$ and $n_{G_{x}} / n_{G}$, for $x=100 \%$ and $x=95 \%$.

Comparing the topology. Apart from the MSA, an interesting information about the selection is the topology of the union of the balls of the conformers selected. The boundary of the union of conformers defines a compact orientable surface, possibly non connected-as the union of conformers may isolate one or several hole(s). By the theorem of classification of connected compact orientable surfaces [28], each such connected component is a sphere with a number $g \geq 0$ of handles attached: for example, the sphere, one-torus, two-torus respectively correspond to $g=0, g=1, g=2$. To characterize these situations, one resorts to Betti numbers, which are respectively $\beta_{0}=1, \beta_{1}=2 g, \beta_{2}=1$. Alternatively, one can compute the Euler characteristic of the surface, that is $\chi=\beta_{0}-\beta_{1}+\beta_{2}=2-2 g$, with $g$ the genus of the surface. Fig. 6 presents and example selection of $g+1$ conformers anchored at the loops extremities, and 
defining a genus $g$ surface ( $g=2$ here). We shall compare the variation of $\beta_{1}$ for $n_{G_{100 \%}}$ conformers selected by algorithm Greedy and HClust.

Comparing the $C_{\alpha}$-rmsd. The measures just described are somewhat tailored to our selection algorithm, since Greedy aims at optimizing the MSA. To provide a fair comparison, we thus also report on a measure based upon the $C_{\alpha}$-rmsd used by HClust. More precisely, to make an assessment on the diversity of a given selection, we investigate the range spanned by the $C_{\alpha}$-rmsd of loops from this selection with respect to the native cocrystallized loop. Notice that since the $C_{\alpha}$ carbons are common to an atomic model and its coarse representation (see beginning of section 3.1), algorithm HClust reports the same selection for the atomic and coarse models, while algorithm Greedy reports two different such selections.

\subsection{Results}

Comparing MSA. In the following, we refer to Tables 3 and 4 in appendix C.2. Speaking of the max values $R_{G}$ and $R_{H}$, one observes that Greedy yields an increase in the range $9-13 \%$ for (Direx, atomic), $11-15 \%$ for (Direx, coarse), $14-54 \%$ for (Loopy, atomic) and $25-56 \%$ for (Loopy, coarse). More interesting is the speed at which the methods peak, as can be seen from the ratios $n_{H_{x}} / n_{G}$ and $n_{G_{x}} / n_{G}$, for $x=100 \%$. The number of conformers required by algorithm Greedy to match the maximum of algorithm HClust incurs a dramatic $k$-fold reduction, where $k$ spans the following ranges (decimals omitted): 9-154 (Direx, atomic), 1-160 (Direx, coarse), 4-79 (Loopy, atomic), 10-79 (Loopy, coarse). On the other hand, as can be seen from the plot Fig. 7 (a typical one), the asymptote is reached rather fast for all algorithms. Focusing on $95 \%$ of the max MSA obtained, the ratios $n_{H_{95}} / n_{G}$ now span the following ranges: 1-6 (Direx, atomic), 1-3 (Direx, coarse), 3-28 (Loopy, atomic), 3-11 (Loopy, coarse). These values call for two conclusions.

First, consider the variation of the ratio $\left(n_{H_{100 \%}} / n_{G}\right) /\left(n_{H_{95} \%} / n_{G}\right)$ for the Direx and Loopy data sets. This ratio is clearly much higher for Direx than Loopy, which has the following explanation: for a dense data set such as Direx, algorithm HClust selects pretty fast good representatives accounting for most of the MSA (95\% here); but further selections fail at significantly increasing the MSA, as seen from much higher ratios $n_{H_{100 \%}} / n_{G}$. On the other hand, algorithm Greedy consistently selects the conformers optimizing the increase of MSA. Second, focus on the statistic $n_{H_{95 \%}} / n_{G}$ for the Direx and Loopy data sets. This ratio is much higher for the latter data set, which shows that algorithm Greedy is also better at selecting large increments of MSA within data sets of conformers exploring more space.

Variations of Betti numbers. For a qualitative expla- nation of these facts ${ }^{1}$, consider the variation of the first Betti number $\beta_{1}$ for the two algorithms. As seen from Tables 5 and 6 in appendix C.2, the selection obtained with algorithm Greedy, when compared to that obtained with HClust, typically features an average value of $\beta_{1}$ which is about 12 times higher for Direx and 5 times higher for Loopy.

The variation of $\beta_{1}$ is illustrated on Fig. 8 , which is also a prototypical plot. Indeed, all such curves feature a sharp peak, followed by a plateau, and algorithm Greedy outperforms its contenders in both regimes. The sharp rise at the beginning of the selection process corresponds to the choice of independent conformers i.e. conformers that do not overlap excepted at their extremities. Such conformers minimize the overlap between balls-in agreement with the criterion targeted by algorithm Greedy. Once the maximum has been reached, the conformers selected bridge gaps, whence a decrease in $\beta_{1}$. The sharp decrease stops as soon as the union of the selection is essentially a topological ball. The union still features small handles. Such handles get created and destroyed upon addition of new conformers, whence the minute fluctuations about the horizontal asymptote of the graphs displaying the variation of $\beta_{1}$.

The variation of $\beta_{1}$ (see plots in appendix C.3) also sheds an interesting light on the relative flexibility of the four loops. As for the MSA variation, the curve of 3 HHR clearly shows that the $n=500$ conformers are not enough in the Loopy data set. We also speculate that a comparison between the maximum value of $\beta_{1}$ obtained and the ensuing plateau encode interesting features on hinges found in the structure. To confirm these statements, though, one would need to geometrically qualify the geometry of the handles defining a basis of the homology groups.

Comparing the $C_{\alpha}$-rmsd. For a given selection output by Greedy (HClust), let $\delta_{G}\left(\delta_{H}\right)$ be the range of $C_{\alpha}$ rmsd spanned by the conformers from the selection with respect to the native bound loop. As reported in appendix on Tables 7 and 8, for models 1BTH, 1CGI and $1 \mathrm{OAZ}$, the ratio $\left(\delta_{G}-\delta_{H}\right) / \delta_{H}$ spans the range [$0.07,0.63]$ when Greedy is run on atomic models, and $[-0.13,0.76]$ if greedy is run on coarse models. Thus, apart from occasional minor losses, Greedy outperforms HClust regardless of the data set and of the representation level, even though the $C_{\alpha}$-rmsd is not the criterion targeted. For 3HHR for the Loopy data set, while the two algorithms perform almost equally for the Direx data set, Greedy is clearly outperformed for the Loopy one. This owes to the length of the loops and its flexibility, and shows the independence of the MSA and $C_{\alpha}$-rmsd

1. The analysis is qualitative for the following reason: a handle accounts for one unit in the $\beta_{1}$ number, whatever its size. That is a large handle coming from a whole loop (as on Fig. 6) has the same weight as a small one coming from the creation of a local cycle between atoms of say a side-chain and the backbone. While computing the Betti numbers is by now standard-we use the $\alpha$-shapes based algorithm of [29]. The calculation of a geometrically pleasant basis of the homology groups is still an active area of research [30]. 


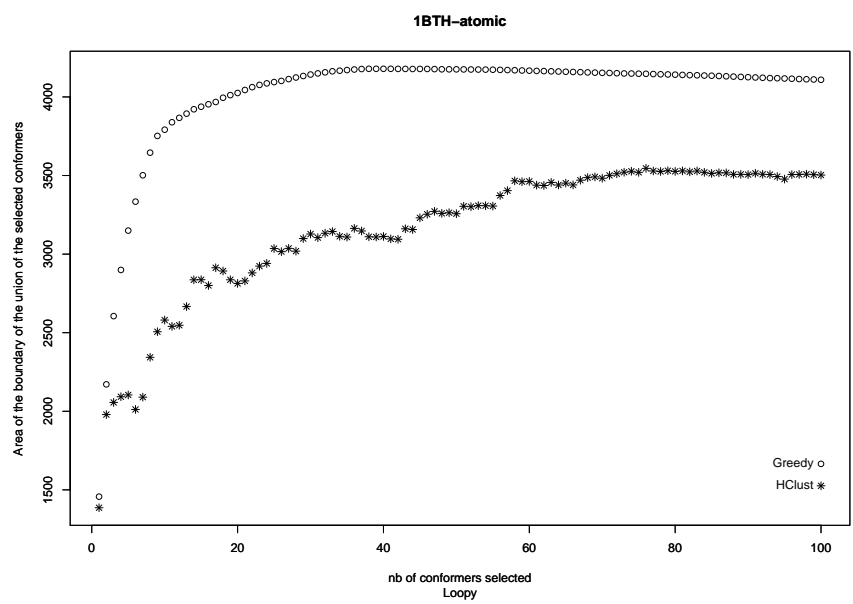

Fig. 7. Loopy data set. Selections of increasing size for 1BTH-atomic: variation of MSA.

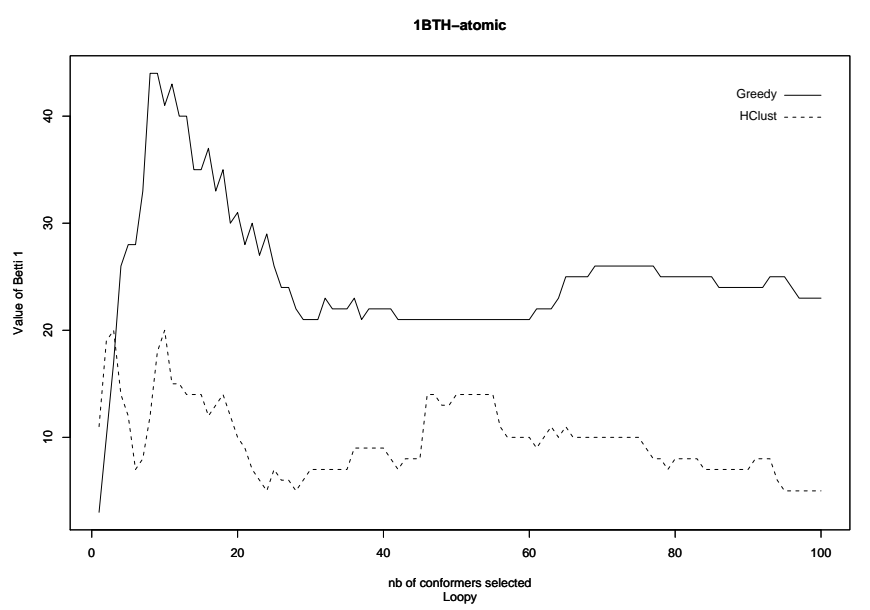

Fig. 8. Loopy data set. Selections of increasing size for 1BTH-atomic: variation of the Betti number $\beta_{1}$.

criteria on this kind of system.

\section{Diverse Ensembles: Docking Assess- MENT}

In this section, report docking results for $1 \mathrm{BTH}, 1 \mathrm{CGI}$ and 1OAZ, based on selections of coarse conformers provided by algorithms HClust or Greedy. Following the discussion in section 4 , we focus on conformer pools generated by Loopy, which are more diverse, and we omit complex $3 \mathrm{HHR}$, since generating a representative pool of conformers for its long flexible loops of 26 amino is a problem in itself.

\subsection{Docking Protocols}

Specifying the ligand and the receptor. To validate the conformer selection strategy based upon MSA maximization, we ran docking simulations on three complexes. Each complex was decomposed into one rigid protein called the ligand $(\mathrm{L})$, and one flexible called the receptor. The receptor itself decomposes into a rigid template (R) and a flexible loop (F). While performing flexible protein docking with conformer ensembles, the strategy consists of using a conformer ensemble for the flexible loop F, this ensemble being selected from a larger pool. Thus, specifying a docking protocol requires specifying the triple $\mathrm{R} / \mathrm{L} / \mathrm{F}$.

To see how, recall that a binary complex used for docking validation features two molecules which have been crystallized under two forms: on their own, i.e. the unbound forms, and in complex i.e. the bound forms. Thus, to specify the rigid parts ( $\mathrm{R}$ and $\mathrm{L}$ ), we provide a tag indicating the origin of the partner, namely $U$ for Unbound and B for Bound. To specify the ensemble associated to $\mathrm{F}$, we provide three pieces of informations: (i) the bound/unbound tag which indicates the loop geometry used to generate the pool of conformers (ii) the selection size, and (iii) the algorithm used to select the conformers from this pool (HClust or Greedy here). For example, F=B-Greedy-10 refers to 10 conformers selected by algorithm Greedy, out of a pool of conformers generated from the Bound structure of the receptor. As a second example, $\mathrm{F}=\mathrm{B}-1$ means that a single loop has been used, the Bound one.

To summarize, we report on the following six docking protocols: three using the Bound form of the receptor, namely B/B/B-1, B/B/B-HClust-10, B/B/B-Greedy10 ; and three using the Unbound form of the receptor, namely U/B/B-1, U/B/B-HClust-10, U/B/B-Greedy10.

Two comments on these protocols are in order. First, notice that the incentive for using the Bound conformation of the flexible region to generate the conformers is the following: for very flexible systems, such as 1CGI mentioned in section 3.1, the reconstruction of the unbound conformation of the flexible loop from the crystallographic data is not possible. (If the conformation of the loop changes across the crystallographic units, the signal is not strong enough for the reconstruction to be carried out.) Second, the particular protocols B/B/B1 and $U / B / B-1$ can be seen as sanity checks, since in using a single loop conformer which is the native one, one expects the docking process to yield satisfactorily putative complexes.

About the pool size and the number of conformers. For each flexible loop, a pool of $n=500$ conformers was generated using Loopy [31], from which $s=10$ were selected using the Greedy and HClust algorithms. Following [5], the choice of $s=10$ comes from a tradeoff between the requirement to have a representative selection, and the computational resources required. In passing, as seen from Table 1, we observe that for all systems but 3HHR, the MSA of the union of the first 10 conformers selected by Greedy realizes more than $80 \%$ of the maximum MSA observed along the iterative greedy selection up to $n$ conformers. Quite clearly, the same table shows that a mere 10 conformers is not enough to represent the flexible loop of $3 \mathrm{HHR}$. 


\begin{tabular}{|c|c||c|c|}
\hline Loopy & $\%$ & Direx & $\%$ \\
\hline 1BTH-atomic & 90.73 & 1BTH-atomic & 99.33 \\
\hline 1CGI-atomic & 95.73 & 1CGI-atomic & 100 \\
\hline 1OAZ-atomic & 82.85 & 1OAZ-atomic & 99.77 \\
\hline 3HHR-atomic & 66.46 & 3HHR-atomic & 99.74 \\
\hline \hline 1BTH-coarse & 87.72 & 1BTH-coarse & 98.88 \\
\hline 1CGI-coarse & 91.23 & 1CGI-coarse & 99.7 \\
\hline 1OAZ-coarse & 83.12 & 1OAZ-coarse & 99.44 \\
\hline 3HHR-coarse & 55.03 & 3HHR-coarse & 99.99 \\
\hline
\end{tabular}

TABLE 1

Percentage of the maximum MSA achieved by Greedy realized by the selection of only ten loops.

\subsection{Initial Conditions for a Protocol}

For a given protocol, we ran $N_{t}$ docking tests using algorithm ATTRACT [17], which is based on the coarse protein representation recalled in section 3.1. This algorithm has been adapted to handle multiple copies of a flexible loop in [5]. In this scheme, using Boltzmann's principle, each copy is assigned a fitness score (between 0 and 1) based upon its interaction energy with the receptor. Each docking test corresponds to a specific position and orientation of the ligand with respect to the receptor. Given these initial conditions, ATTRACT performs a sequence of minimizations so as to explore the six degrees of freedom of the ligand. At each stage, the energy of each conformation of the complex is computed. Upon termination, the loop selected is that having the highest fitness score. An assessment of the quality of the proposed complex is then based upon two figures: (i) the interaction potential energy $E$ of the complex (ii) the I-rmsd of the atoms of the ligand.

For the $N_{t}$ tests associated to a given protocol, the plot of the pairs (E,I-rmsd) defines the energy landscape of the docking experiment. Thus, a conformer ensemble is satisfactory if the landscape features at least one conformer yielding a large number of points $(E, \mathrm{I}-$ rmsd) next to the bottom left corner of the energy landscape. Practically, we represent an energy landscape using buckets. For a given bucket $B_{i}$ and conformer $C_{j}$, let $s_{i, j}$ be the number of times conformer $C_{j}$ yields a complex whose energy and I-rmsd fall in bucket $B_{i}$. (Notice that $\sum_{i, j} s_{i, j}=N_{t}$.) Finally, for a given bucket $B_{i}$, denote $l_{i}$ the index of the conformer that yields the largest value of $s_{i, j}$, and let $r_{i}=\sum_{j=1, \ldots, n ; j \neq l_{i}} s_{i, j}$. In bucket $B_{i}$ we display the score $s_{i, l_{i}}$, together with $r_{i}$ when $r_{i} \neq 0$. The color used to do so is that associated to conformer $l_{i}$, with one color per conformer.

\subsection{Results}

For each selection method, a total of $N_{t} \sim 35,000$ docking tests were run using the same $s=10$ selected conformers. To analyse the results, we plot on the portion of the energy landscape corresponding to a I-rmsd $\leq 15 \AA$ with a negative energy. An example of such a plot is presented on Fig. 9, and we refer the reader to appendix C.4 for the remaining plots. In analyzing a landscape and since the docking process is a coarse one, we just aim at identifying conformers with good potentiality for an atomic docking process. We thus skip a detailed atomic discussion of the results, a notoriously difficult task [32]. For six docking protocols examined (three systems, Bound and Unbound receptors for each), we argue that the results decompose as follows: three favorable to Greedy, two ties, one favorable to HClust.

Docking improved using Greedy. For complex 1BTH, the docking protocol $\mathrm{B} / \mathrm{B} / \mathrm{B}-\mathrm{Greedy}-10$ leads to 161 predictions with I-rmsd $\in(1 ; 2]$ and energy below -21 units. Only 3 such predictions are found using docking protocol B/B/B-HClust-10. See Fig. 9. The same kind of result can be observed when using the unbound form of the receptor of $1 \mathrm{CGI}$. Indeed, U/B/B-Greedy-10 leads to 160 predictions with I-rmsd $\in(3 ; 4]$ and energy below -15 units, while $\mathrm{U} / \mathrm{B} / \mathrm{B}-\mathrm{HCl}$ ust-10 yields 18 such predictions. For complex 10AZ, neither U/B/B-Greedy10 nor U/B/B-HClust-10 leads to a high number of predictions below $5 \AA$ I-rmsd and below -15 energy units: 5 with one loop, and 13 with 5 different loops respectively. But considering predictions with I-rmsd in interval $(5,7]$ and energy below -15 units, U/B/BGreedy-10 leads to 195 predictions with the same loop while U/B/B-HClust-10 leads to one such prediction.

Tie between Greedy and HClust. The results of the docking involving the unbound form of the receptor of the complex $1 \mathrm{BTH}$ are more mitigated. U/B/B-HClust10 leads to two predictions with the same loop with I-rmsd below $5 \AA$ and below -15 energy units, while U/B/B-Greedy-10 leads to no such prediction. When considering predictions with I-rmsd in interval $(5,7]$ and energy below -15 energy units, both U/B/B-Greedy10 and U/B/B-HClust-10 lead to more than 300 such predictions. The results of the docking involving the bound form of the receptor of the complex 10AZ needs further scrutiny to detect whether some improvement is achieved by B/B/B-Greedy-10 compared to U/B/BHClust-10. Indeed, no highly populated region with low energy and low I-rmsd clearly emerges.

No improvement while using Greedy. The results of the docking involving the bound form of the receptor of the complex 1CGI are more favorable to the docking protocol $\mathrm{B} / \mathrm{B} / \mathrm{B}-\mathrm{HCl}$ ust-10. Nevertheless, it must be noticed that even if $\mathrm{B} / \mathrm{B} / \mathrm{B}-\mathrm{HCl}$ ust-10 leads to a larger number of good predictions, the energy of these predictions is much higher than those obtained with the native loop in the protocol $\mathrm{B} / \mathrm{B} / \mathrm{B}-1$.

\subsection{Running Times}

Having discussed the docking results, some comments are in order regarding the computational cost of the algorithms. The naive and priority based selection algorithms were run on a PC computer equipped with a Xeon processor (quadcore) at $2.33 \mathrm{GHz}$, and 16GB of RAM. Quite surprisingly, we observed a factor of one order of magnitude in favor of the naive implementation, and 
1BTH B/B/B-HClust-10

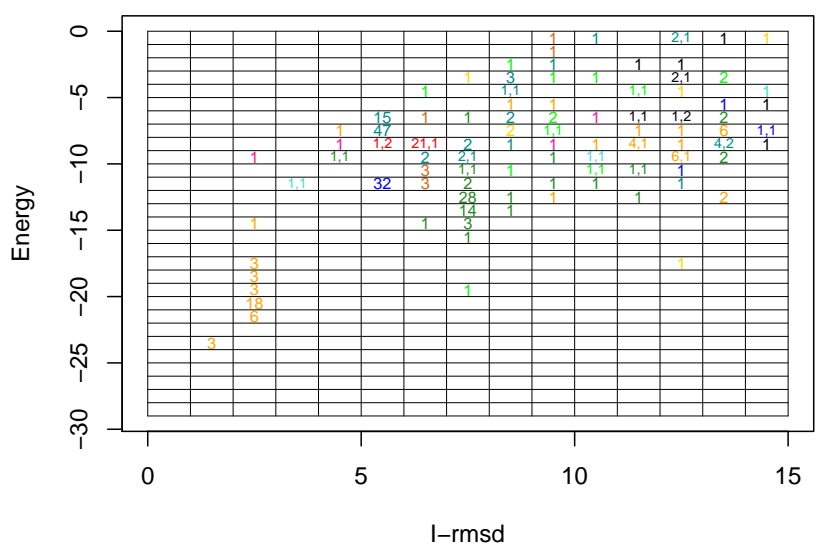

1BTH B/B/B-Greedy-10

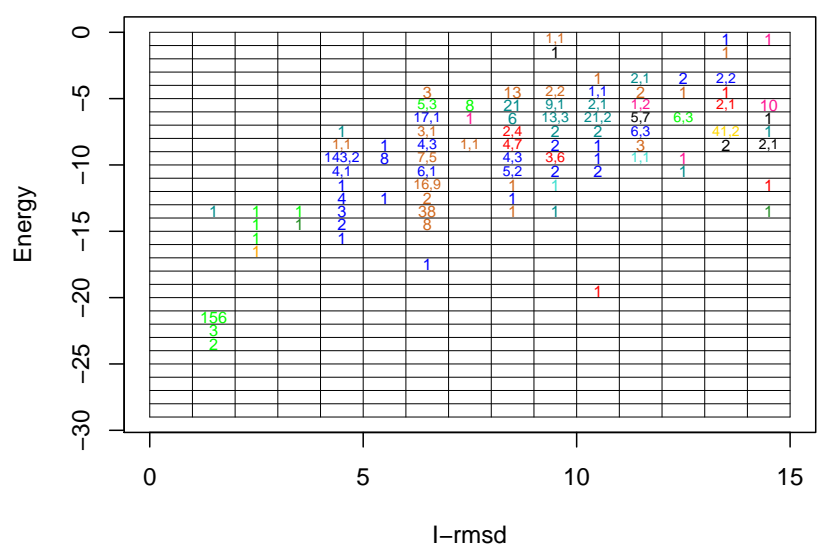

Fig. 9. 1BTH: binning the docking tests using the Bound form of the receptor. The color associated to a bucket is associated to the loop yielding the highest score in this bucket. A highly populated region next to the bottom left corner of the plot indicates that a satisfactory conformer was present. See text for details.

in particular when $s$ is much smaller than $n$. Although asymptotically optimal, the problem of the priority based algorithm lies in the computation of the arrangement: for $n$ conformers, the size of the arrangement on a given ball may be (and actually is on some examples) as high as $n^{2}$. Using the naive implementation, the selection of ten loops requires about half an hour (respectively about five minutes) using the all atoms representation (respectively using the coarse grain representation).

As reported in [5], the docking algorithm using ten conformers requires 31 hours on a $2.2 \mathrm{GHz}$ Athlon PC.

\section{Conclusion}

Summary of results. For systems whose flexibility cannot be explored resorting to molecular dynamics simulations, the manipulation of discrete ensemble of pre- generated conformers is the route of choice. This strategy is valid for fragments of any size, namely for side chains, protein loops or domains. Because the generation of such ensembles does not take into account the whole environment of the fragment (in the whole protein or complex), the energetic functionals used to compute the energy of a conformer cannot, in general, be directly related to the thermodynamic equilibrium between the conformations. This observation calls for the development of methods providing a rather uniform sampling of the conformational space of the fragment considered, so as to retain conformers avoiding obvious steric clashes. But such algorithms face one central difficulty: that of characterizing the conformational space coverage, so as to maximize the diversity of the conformers. In this context, we make three contributions.

First, we present geometric optimization methods geared towards the characterization and the selection of conformational diversity. Given a collection of conformers, the methods aim at returning a selection maximizing a functional of the volume occupied by the conformers, or of the molecular surface exposed by the conformers. Greedy strategies are used to solve these problems, and theoretical bounds are proved.

Second, for the particular problem of the optimization of the MSA, we make a geometric assessment of the conformational diversity of the conformers selected, based upon experiments carried out on four flexible protein loops. We show that our greedy strategy matches the MSA of standard selection methods, using, depending on the particular system and the model (atomic or coarse), a number of conformers between one and two orders of magnitude smaller. Moreover, tracking the variation of the MSA together with topological informations of the selection (the Betti numbers) yields insights on the quality of the coverage of the conformational space associated to a collection of conformers.

Third, using coarse representations of three of these protein models, we compare the results of a multi-copy docking algorithm, for two sets of copies: one selected by our greedy strategy-Greedy, and one generated by a standard hierarchical clustering algorithm-HClust. For six docking protocols (three systems, Bound and Unbound receptors for each), the results decompose as follows: three favorable to Greedy, two ties, one favorable to HClust.

Applications and outlook. Our developments have a number of direct applications. First, our characterization of the conformational diversity based upon geometric and topological measures, together with the greedy strategy, should prove useful to improve the conformational space coverage of conformer generation methods. For example, algorithms Loopy and Direx could bootstrap on our selections so as to improve their conformational diversity. Second, the positive results obtained for coarse docking call for further developments. In particular, bootstrapping on the selections of coarse conformers generated by Greedy so as to generate high-quality 
atomic models should improve the predictions for challenging flexible protein-protein complexes.

Interestingly, our work also raises a number of open theoretical questions. First, for a particular problem (conformer generation, docking), the question of the particular functional to be optimized (volume based, surface based) needs to be addressed. Volume based and surface based are obvious candidates, especially since the surface exposed by a collection of balls is the geometric locus where interaction occurs. But these might be seen as a first approximations to qualify the conformational diversity. That is, because covering a $3 D$ volume with a collection of conformers does not admit a unique solution, it might actually be necessary to incorporate into the functional some measure of the multiplicity of the cells of the volume or surface arrangements, so as to guarantee that each portion of space is covered the same number of times. Second and from a more algorithmic perspective, while our current running times are comparable to those required by the algorithms exploiting the conformer selections, provably good output sensitive algorithms deserve further investigation.

\section{ACKNOWLEDGMENTS}

The authors wish to thank J. Bernauer, G.F. Schröder, $P$. Yao on the one hand, and F. Nielsen on the other, for insightful discussions about conformer generation methods and optimization, respectively. The molecular illustrations were produced using the software PyMOL [33].

\section{References}

[1] J. Janin and S. Wodak, "The Third CAPRI Assessment Meeting Toronto, Canada, April 20-21, 2007," Structure, vol. 15, no. 7, pp. 755-759, 2007.

[2] J. Monod, J. Wyman, and J. Changeux, "On the nature of allosteric transitions: a plausible model," Journal Molecular Biology, vol. 12, pp. 88-118, 1965.

[3] R. Grunberg, J. Leckner, and M. Nilges, "Complementarity of structure ensembles in protein-protein binding," Structure, vol. 12, pp. 2125-2136, 2004.

[4] A. Canutescu, A. A. Shelenkov, and R. Dunbrack, "A graph theory algorithm for protein side-chain prediction," Protein Science, vol. 12, pp. 2001-2014, 2003.

[5] K. Bastard, C. Prévost, and M. Zacharias, "Accounting for loop flexibility during protein-protein docking," Proteins, vol. 62, no. 4, pp. 956-969, 2006.

[6] J. Cortés, T. Siméon, V. Ruiz de Angulo, D. Guieysse, M. RemaudSiméon, and V. Tran, "A path planning approach for computing large-amplitude motions of flexible molecules," Bioinformatics, vol. 21, pp. 116-125, 2005.

[7] A. Dhanik, P. Yao, N. Marz, R. Propper, C. Kou, G. Liu, H. van den Bedem, and J. Latombe, "Efficient algorithms to explore conformation spaces of flexible protein loops," in 7th Workshop on Algorithms in Bioinformatics (WABI), Philadelphia, 2007, pp. 265-276.

[8] A. Malevanets, F. Sirota-Leite, and S. Wodak, "Mechanism and energy landscape of domain swapping in the B1 domain of protein G," Journal of Molecular Biology, vol. To appear, 2008.

[9] M. R. Garey and D. S. Johnson, Computers and Intractability: A Guide to the Theory of NP-Completeness. New York, NY: W. H. Freeman, 1979

[10] U. Feige, "A threshold of $\ln \mathrm{n}$ for approximating set cover," Journal of the ACM, vol. 45, no. 4, pp. 634-652, 1998. [Online]. Available: citeseer.ist.psu.edu/article/feige98threshold.html
[11] P. Fishburn and W. Gehrlein, "Pick-and choose heuristics for partial set covering," Discrete Applied Mathematics, vol. 22, no. 2, pp. 119-132, 1989.

[12] G. Cornuejols, M. Fisher, and G. Nemhauser, "Location of bank accounts to optimize float: An analytic study of exact and approximate algorithms," Management Science, vol. 23, no. 8, pp. 789-810, 1977.

[13] G. Nemhauser, L. Wolsey, and M. Fisher, "An analysis of approximations for maximizing submodular set functions," Mathematical Programming, vol. 14, no. 1, pp. 265-294, 1978.

[14] F. Cazals and S. Loriot, "Computing the exact arrangement of circles on a sphere, with applications in structural biology," in Proc. 23th Annual Symposium Computational Geometry-Video/Multimedia track, 2007.

[15] — "Computing the arrangement of circles on a sphere, with applications in structural biology," Computational Geometry : Theory and Applications, Under revision. [Online]. Available: https://hal.inria.fr/inria-00118781

[16] P. M. M. de Castro, F. Cazals, S. Loriot, and M. Teillaud, "Design of the CGAL spherical kernel and application to arrangements of circles on a sphere," Computational Geometry : Theory and Applications, Under revision. [Online]. Available: https://hal.inria.fr/inria-00173124

[17] M. Zacharias, "Protein-protein docking with a reduced protein model accounting for side-chain flexibility," Protein Science, vol. 12, no. 6, pp. 1271-1282, 2003.

[18] M. Lensink, R. Mendez, and S. Wodak, "Docking and scoring protein complexes: CAPRI 3rd Edition." Proteins, vol. 69, no. 4, pp. 704-18, 2007.

[19] R. Chen, J. Mintseris, J. Janin, and Z. Weng, "A protein-protein docking benchmark," Proteins, vol. 52, no. 1, pp. 88-91, 2003.

[20] L. James, P. Roversi, and D. Tawfik, "Antibody multispecificity mediated by conformational diversity," Science, vol. 2999, p. 1362 1367, 2003.

[21] Z. Xiang, C. Soto, and B. Honig, "Evaluating conformational free energies: The colony energy and its application to the problem of loop prediction," Proceedings of the National Academy of Sciences, vol. 99 , no. 11 , p. 7432,2002

[22] K. Noonan, D. O'Brien, and J. Snoeyink, "Probik: Protein Backbone Motion by Inverse Kinematics," The International Journal of Robotics Research, vol. 24, no. 11, p. 971, 2005.

[23] G. Schröder, A. Brunger, and M. Levitt, "Combining efficient conformational sampling with a deformable elastic network model facilitates structure refinement at low resolution," Structure, vol. 15, pp. 1630-1641, 2007.

[24] A. Fiser, R. Do, and A. Šali, "Modeling of loops in protein structures," Protein science, vol. 9, no. 09, pp. 1753-1773, 2000.

[25] E. Lindahl, B. Hess, and D. van der Spoel, "GROMACS 3.0: a package for molecular simulation and trajectory analysis," Journal of Molecular Modeling, vol. 7, no. 8, pp. 306-317, 2001.

[26] "CGAL, Computational Geometry Algorithms Library," http:// www.cgal.org.

[27] A. Gordon, Classification. Chapman \& Hall/CRC, 1999.

[28] M. Henle, A Combinatorial Introduction to Topology. Dover, 1994

[29] C. Delfinado and H. Edelsbrunner, "An incremental algorithm for Betti numbers of simplicial complexes on the 3-sphere," Computer Aided Geometric Design, vol. 12, no. 7, pp. 771-784, 1995.

[30] C. Chen and D. Freedman, "Quantifying homology classes ii: Localization and stability," Preprint, 2007, arXiv:0709.2512v2.

[31] Z. Xiang, C. S. Soto, and B. Honig, "Evaluating conformational free energies: The colony energy and its application to the problem of loop prediction," Proceedings of the National Academy of Sciences, vol. 99, no. 11, pp. 7432-7437, 2002.

[32] N. London and O. Schueler-Furman, "Funnel Hunting in a Rough Terrain: Learning and Discriminating Native Energy Funnels," Structure, vol. 16, no. 2, pp. 269-279, 2008.

[33] D. W.L., "The PyMOL molecular graphics system," http://www. pymol.org.

[34] N. Akkiraju and H. Edelsbrunner, "Triangulating the surface of a molecule," Discrete Applied Mathematics, vol. 71, no. 1-3, pp. 5-22, 1996.

[35] B. de Groot, D. van Aalten, R. Scheek, A. Amadei, G. Vriend, and $\mathrm{H}$. Berendsen, "Prediction of protein conformational freedom from distance constraints," Proteins Structure Function and Genetics, vol. 29, no. 2, pp. 240-251, 1997. 


\section{APPENDIX A}

\section{SUPPLEMENT: VOLUMETRIC DECOMPOSITIONS}

\section{A.1 Greedy: Approximation Factor and Optimality}

\section{A.1.1 Approximation Factor}

We shall use the following notations. The conformer selected at the $k^{\text {th }}$ step is denoted $C_{k}$, and the weight of the optimum set of conformers $O P T$. Also, let us denote by $w^{*}\left(C_{k}\right)$ as the sum of the weights of the new elements in $C_{k}$ that have not been covered in $C_{j}, 1 \leq j<k$. We need the following lemma in order to prove the theorem.

Lemma A.1. For $1 \leq k \leq s$, the following holds:

$$
w^{*}\left(C_{k}\right)+\frac{1}{s} \sum_{j=1}^{k-1} w^{*}\left(C_{j}\right) \geq \frac{O P T}{s} .
$$

Proof: At the $k^{\text {th }}$ step, we select $C_{k}$ that maximizes the weight of the new elements $A_{i}$ being covered. The weight of the elements that are covered by the optimum solution but not yet covered by the $(k-1)$ is at least

$$
O P T-\sum_{j=1}^{k-1} w^{*}\left(C_{j}\right)
$$

Since $w$ is non-negative, the union-bound property states that for any collection of conformers $C_{1}, \ldots, C_{p}$, one has $w\left(C_{1} \cup \cdots \cup C_{p}\right) \leq \sum_{i=1, \ldots, p} w\left(C_{i}\right)$. Since all the elements involved in Eq. (6) are covered by the optimum set of conformers, by the union-bound property, there must exist a conformer that covers new elements with total weight at least

$$
\frac{1}{s}\left(O P T-\sum_{j=1}^{k-1} w^{*}\left(C_{j}\right)\right) .
$$

Since $C_{k}$ maximizes the weight of the new elements being covered, we must have

$$
w^{*}\left(C_{k}\right) \geq \frac{1}{s}\left(O P T-\sum_{j=1}^{k-1} w^{*}\left(C_{j}\right)\right) .
$$

Rearranging completes the claim.

Remark. The non-negativity assumption is critical in the proof of Lemma A.1. As a counter-example, consider the sets $C_{1}=\{e 1, e 2\}, C_{2}=\{e 2, e 3\}$ with $w(e 1)=w(e 3)=1$ and $w(e 2)=-1$. The union-bound fails for $w\left(C_{1} \cup C_{2}\right)$.

Using Lemma A.1, the proof of Thm. 2.1 goes as follows:

Proof: Thm. 2.1 Multiplying the inequality obtained for step one by $\left(\frac{s-1}{s}\right)$ and adding to the inequality for step two, we get

$$
w^{*}\left(C_{1}\right)+w^{*}\left(C_{2}\right) \geq\left(1+\left(\frac{s-1}{s}\right)\right) \frac{O P T}{s}
$$

We multiply this equation again by $\left(\frac{s-1}{s}\right)$ and add to the equation for step three, and so on. We get the following,

$$
\sum_{j=1}^{k} w^{*}\left(C_{j}\right) \geq\left(1-\left(\frac{s-1}{s}\right)^{k}\right) O P T
$$

For $k=s$, we get,

$$
\frac{\sum_{j=1}^{s} w^{*}\left(C_{j}\right)}{O P T} \geq\left(1-\left(\frac{s-1}{s}\right)^{s}\right)
$$

The left hand side is the ratio of the weight of the subset of $\mathcal{C}$ chosen by the greedy approach and the optimum solution i.e. that approximation factor and hence we have the above theorem. The fact that the above ratio is greater than $1-\frac{1}{e}$ for all $s$ is a trivial exercise.

\section{A.1.2 Optimality}

To prove Thm. 2.1, we construct tight examples for the greedy approach.

Proof: Thm. 2.1 Fix a given $s$. We shall construct an example where the greedy approach can achieve an approximation ratio arbitrarily close to $1-\left(1-\frac{1}{s}\right)^{s}$.

Let

$$
\begin{gathered}
\mathcal{A}=\left\{A_{i}\right\}_{i=1, \ldots,\left(s^{2}+s\right)} \\
\forall i, j \text { s.t. } 0 \leq i<s, 1 \leq j \leq s, w\left(A_{i . s+j}\right)=\frac{1}{s^{2}}\left(\frac{s-1}{s}\right)^{i} \\
\forall j \text { s.t. } 1<j \leq s, w\left(A_{s^{2}+j}\right)=\frac{1}{s}\left(\frac{s-1}{s}\right)^{s}-\epsilon
\end{gathered}
$$

The conformers are defined as follows

$$
\mathcal{C}=\left\{C_{i}\right\}_{i=1, \ldots, 2 s}
$$

$$
\begin{aligned}
& \forall i \text { s.t. } 1 \leq i \leq s, C_{i}=\bigcup_{j=(i-1) . s+1}^{i . s} A_{j} \\
& \forall i \text { s.t. } \leq i \leq s, C_{s+i}=\bigcup_{j \equiv i(\bmod s)} A_{j}
\end{aligned}
$$

Simple calculations lead us the following total weights for the conformers

$$
\begin{gathered}
\forall 1 \leq i \leq s, w\left(C_{i}\right)=\frac{1}{s}\left(\frac{s-1}{s}\right)^{i-1} \\
\forall 1 \leq i \leq s, w\left(C_{s+i}\right)=\frac{1}{s}-\epsilon
\end{gathered}
$$

The optimum choice of $\mathcal{S}$ with $|\mathcal{S}|=s$ is clearly $\left\{C_{i}\right\}_{i=s+1, \ldots, 2 s}$ with total weight $1-s \epsilon$. Whereas the greedy method would choose $\left\{C_{i}\right\}_{i=1, \ldots, s}$, with a maximum weight of $1-\left(1-\frac{1}{s}\right)^{s}$, giving an approximation factor arbitrarily close to $1-\left(1-\frac{1}{s}\right)^{s}$. 


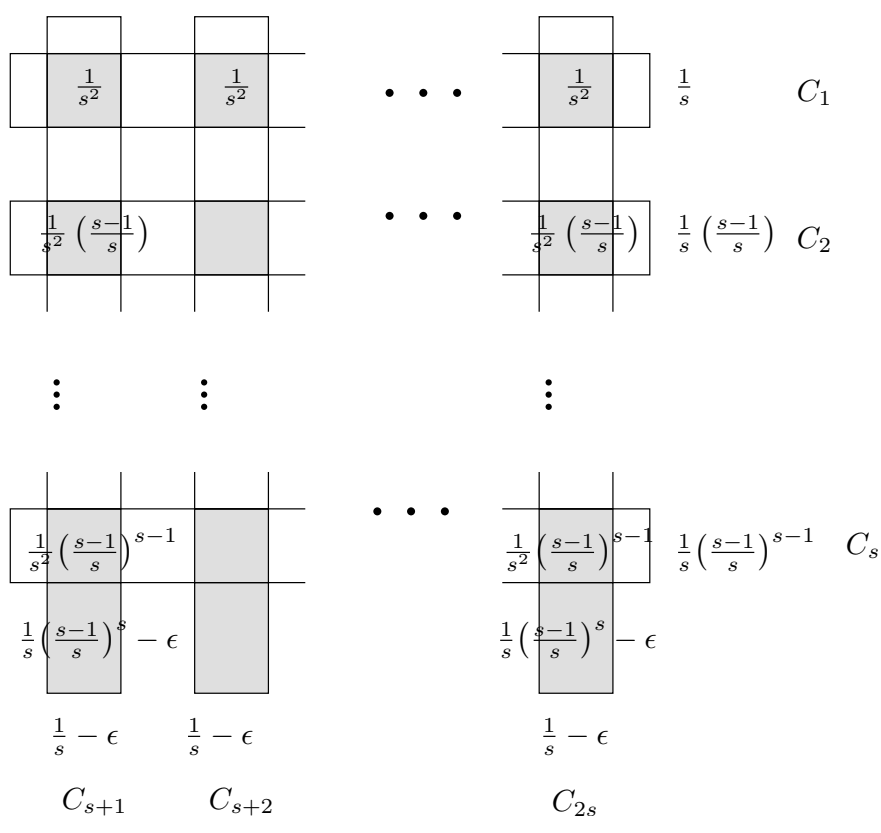

Fig. 10. A tight example for the greedy strategy

\section{APPENDIX B SUPPLEMENT: SURFACE DECOMPOSITIONS}

\section{B.1 Approximating Factor for Problem 2}

The following counter-example sets the approximation ratio for the greedy algorithm for the boundary surface case.

Proof: Observation 1. Consider a large ball $B$, and place $s$ small non-intersecting balls $\left(B_{1}, \ldots, B_{s}\right)$ with their centers on the surface of $B$. The surface of each $B_{i}$ is now divided into 2 patches. To the patch which lies inside $B$, we assign a weight of $s$. To each surface patch of $B$ covered by some $B_{i}$, we assign a weight of $1+\epsilon$. All other surface patches are assigned a weight of 0 .

The greedy strategy would first pick $B$ because it has the largest exposed weight of $s(1+\epsilon)$. Now picking any $s-1$ of the $B_{i}$ 's would leave us with an exposed weight of only $s(1+\epsilon)-(s-1)(1+\epsilon)=1+\epsilon$. On the opposite, a selection of the $s$ smalls balls would have given us total exposed surface weight of $s^{2}$. This approximation factor arbitrarily close to $1 / s^{2}$.

\section{B.2 Naive Algorithm for Surface Arrangement}

Proof: Thm. 3.1 To compute $w\left(G_{i-1} \cup\left\{C_{j}\right\}\right)$, one needs the boundary of the corresponding balls. For a collection of $i$ balls, this is done in worst-case optimal time of $O\left(i^{2}\right)$, by first computing the regular triangulation of the balls, and then by retrieving the boundary of the union from the $\alpha$-complex with $\alpha=0$ [34]. The overall complexity is thus bounded by $\sum_{i=1}^{s}(n-i+1) O\left(i^{2}\right)$, whence the claim.

\section{B.3 Priority-based Algorithm for Surface Arrange- ment}

Notations. If $X$ refers to a collection of conformers, $\cup X$ refers to the domain covered by these conformers, and $\partial \cup X$ refers to the boundary of the union of conformers in $X$. We shall abuse notations, as we shall also use $\partial \cup X$ to refer to the finite number of spherical patches bounding the boundary of the union. Notice though, that the inclusion of a region $r$ in the geometric boundary will be denoted $r \subset \cup X$, while the membership to the finite set describing this boundary will be denoted $r \in \partial \cup X$. Computing the surface decompositions. Using the algorithm of [14], [15], [16], we compute the arrangement on each sphere, induced by the intersection circles with other spheres. The output consists of:

- $D\left(S_{i}\right)=\left\{P_{k}\right\}$ : patches on sphere $S_{i}$,

- $H\left(P_{k}\right)$ : collection of spheres covering patch $P_{k}$,

from which we easily derive:

- $K\left(S_{i}\right)$ : collection of patches covered by sphere $S_{i}$,

- $B\left(C_{i}\right)$ : patches contributing to the boundary of conformer $C_{i}$.

Algorithm. We now present Algorithm 1, which is illustrated on Fig. 11.

Let $G_{i-1}$ be the collection of conformers selected up to stage $i-1$, and denote $C_{s_{i}}$ the $i$ th conformer selected. Also denote $R_{i}$ the candidate conformers remaining once the $i$ th conformer has been selected. In order to select $C_{s_{i}}$, we maintain a priority queue $Q$ such that the key associated to a conformer $C_{l}$ is $k\left(C_{l}\right)=w\left(G_{i-1} \cup\left\{C_{l}\right\}\right)$ $w\left(G_{i-1}\right)$.

Apart from the heap itself, we shall use the following data structures:

- GB: greedy selection boundary, i.e. patches found on $\partial \cup G_{i-1}$,

- $H_{Q}\left(P_{k}\right)$ : candidate conformers covering patch $P_{k}$.

As the arrangement calculation provides us with a list $H\left(P_{k}\right)$ of balls covering a given patch $P_{k}$, the list of conformers $H_{Q}\left(P_{k}\right)$ covering $P_{k}$ is easily set up.

We shall also assume a patch found on the boundary of a candidate conformer has a status with respect to to $G_{i-1}: \operatorname{status}\left(P_{k}\right)=$ covered iff $P_{k} \subset \cup G_{i-1}$, and exposed otherwise. Upon selection of conformer $C_{s_{i}}$, two types of patches have to be taken care of:

$\triangleright$ Case 1: patches covered by $C_{s_{i}}$, which are found either on $\partial \cup G_{i-1}$ (Case 1a), or patches found on the boundary of conformers from $R_{i}$ (Case 1b).

Consider sub-case 1a, i.e. a $P_{k}$ patch found on $\partial \cup G_{i-1}$ which is covered by $C_{s_{i}}$. If this patch is also covered by another conformer $C_{l}$ in $R_{i}$, the weight of this conformer has to be updated as $k\left(C_{l}\right) \leftarrow k\left(C_{l}\right)+w\left(P_{k}\right)$. Indeed, conformers $C_{s_{i}}$ and $C_{l}$ were competing in the queue, and both had been subtracted $w\left(P_{k}\right)$ to compare the relative increments $k\left(C_{s_{i}}\right)$ and $k\left(C_{l}\right)$. Now that $C_{s_{i}}$ has been selected, and since patch $P_{k}$ has already been accounted for in the weight of conformer $C_{s_{i}}$, the weight of conformer $C_{l}$ has to be corrected as indicated.

Consider now sub-case $1 \mathrm{~b}$, i.e. a patch $P_{k}$ found on the boundary of a candidate conformer. This patch being 
now covered by $C_{s_{i}}$, it will not contribute to an increment of the boundary of the union, so that conformer $C_{l}$ has to be updated as $k\left(C_{l}\right) \leftarrow k\left(C_{l}\right)-w\left(P_{k}\right)$.

$\triangleright$ Case 2: patches found on the boundary $\partial \cup G_{i}$ contributed by conformer $C_{s_{i}}$. In selecting the $i+1$ th conformer, such patches may get covered by candidate conformers. The weight of each such conformer $C_{l}$ thus has to be updated as $k\left(C_{l}\right) \leftarrow k\left(C_{l}\right)-w\left(P_{k}\right)$. Note in passing that the fact that several candidates may cover such a patch is responsible for the afore-described subcase 1a.

To prove Thm. 3.2, we shall assume that the priority queue is implemented using a Fibonacci heap, while dictionaries are handles using hash tables. Under these assumptions:

Proof: Thm. 3.2 We first note that each hash-set operation and UpdateKey operation individually takes $O(1)$ amortized time, whereas the RemoveMin operation takes $O(\log n)$ time -using Fibonacci heaps.

The outermost loop and hence the RemoveMin operation is repeated $s$ times. We now look at the calls of Update_H_lists. This function is called at most once for each conformer. The first loop in the function runs at most once for each primitive. For each primitive, the two inner most lines are executed as many times as there are patches that are covered by the primitive. Summing over all possible primitives and conformers, the number of times the inner most lines are executed is clearly bounded by $\tau$. Now, consider the loop that repeats for all patches $P_{k}$ that are covered by the primitive $S_{j}$. The statements inside the if loop are executed if the patch was on the boundary of the union of previously selected conformers. If this is the case, the patch no longer remains on the boundary after the execution of this part. So the lines inside the if part are executed at most once for each patch. In the if part, there is a loop that repeats for every candidate conformer that covers the patch. Summing over all possible patches, these lines are executed at most $\tau$ times. The else part takes constant time in each run, and is repeated for every candidate conformer that contains the patch. Thus, the else part is also repeated at most $\tau$ times. The second loop for the boundary patches repeats at most once for each patch. The inner loop there, again repeats for each candidate that contains the patch, hence the number of executions of the innermost statement is again bounded by $\tau$.

Thus, overall the execution of the algorithm is bounded by $O(\tau+s \log n)$.

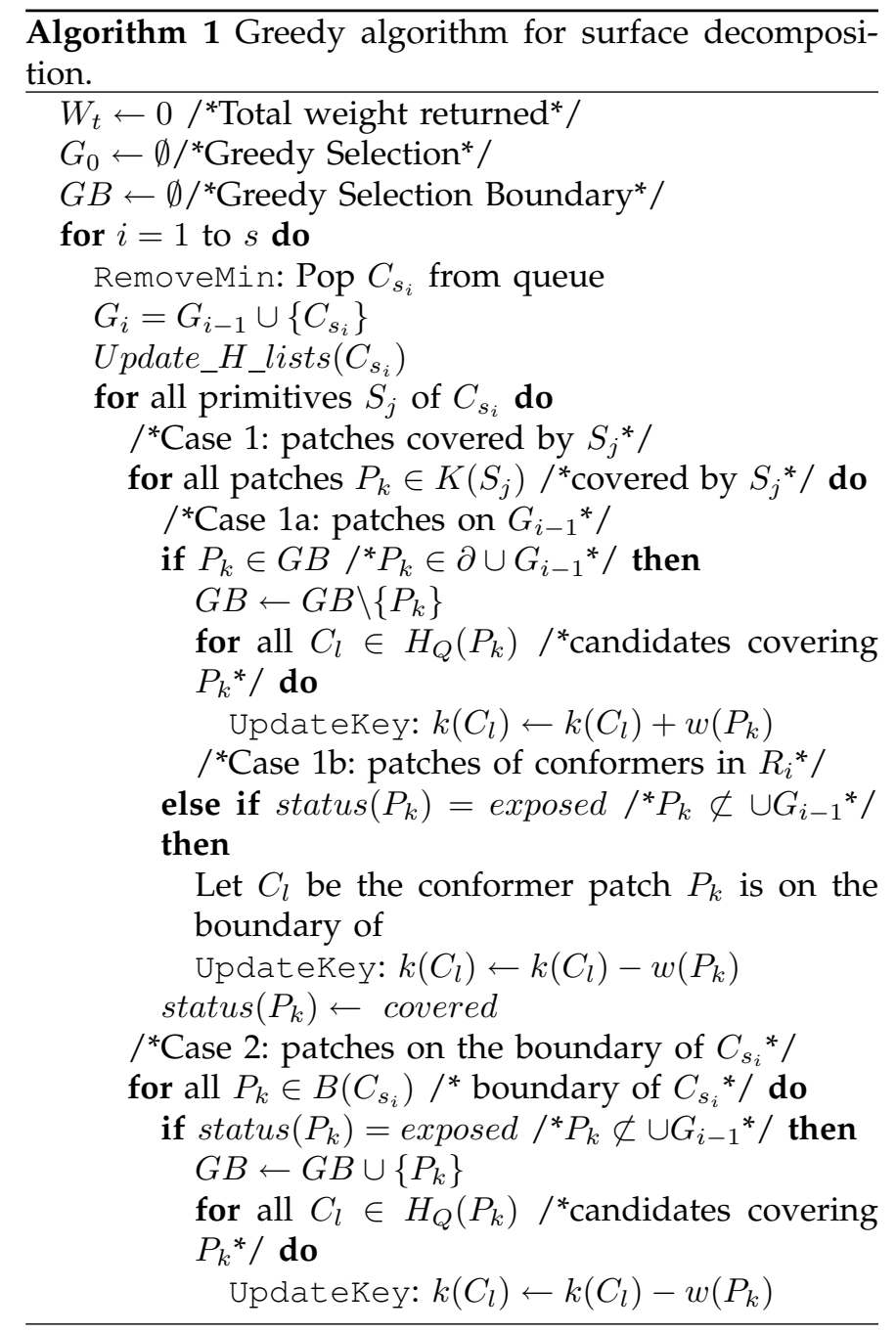

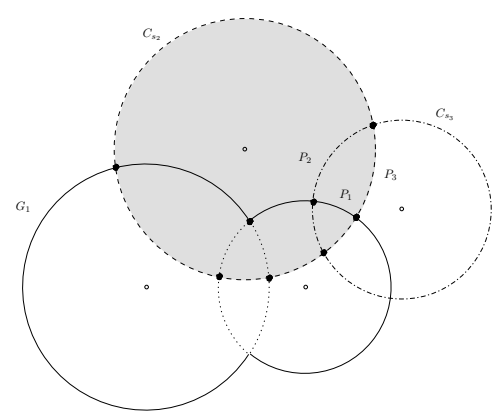

Fig. 11. Greedy algorithm for surface weights. Patches triggering updates of keys upon selection of $C_{s_{2}}$ are $P_{1}, P_{2}, P_{3}$. 


\section{APPENDIX C SuPPlement: Material AND Methods \\ C.1 Direx and Loopy}

We selected two algorithms to generate loop conformers, which respectively yield dense and sparse ensembles of conformers. Algorithm Direx [23], based on algorithm CONCOORD [35], handles a whole protein and processes all the atoms in the same way. The method consists of performing perturbations of the atomic positions while preserving constraints on internal coordinates (bond lengths and dihedral angles). The generation of $n$ conformers is greedy, since the $k$ th conformer is taken as starting point for the generation of the $k+1$ th one. Applying this algorithm to a loop from a PDB structure yields a collection of conformers spanning a relatively small region around the original loop in the PDB file.

Algorithm Loopy [21] is a genetic-like algorithm which consists of evolving a population of loops, the $k+1$ th generation mixing a subset (survivors) of the $k$ th generation together with new individuals derived from this subset. The main features of the algorithm are twofold. First, the algorithm focuses on the backbone, onto which side-chains are added using a rotamer library. Second, the selection of the survivors uses a colony energy. This energy features a potential energy term, together with an entropy term encoding the spread of the neighborhood of a given conformation. This latter term accounts for the usual enthalpy-entropy competition, since a high internal energy conformation might be promoted thanks to a large entropy. This strategy naturally yields rather diverse sets of conformations. 


\begin{tabular}{|c|c|c||c|c||c|}
\hline PDBCODE & MSA Direx & MSA Loopy & Nb balls & Nb res. & RMSD \\
\hline 1BTH-atomic & 1906.01 & 3423.34 & 108 & 12 & $5.7 \AA$ \\
\hline 1BTH-coarse & 1639.72 & 3142.11 & 29 & 12 & \\
\hline 1CGI-atomic & 1867.17 & 3516.97 & 103 & 13 & unres. \\
\hline 1CGI-coarse & 1583.7 & 3032.78 & 28 & 13 & \\
\hline 1OAZ-atomic & 2535.6 & 4788.63 & 142 & 14 & $2.1 \AA$ \\
\hline 1OAZ-coarse & 2262.51 & 4211.13 & 37 & 14 & \\
\hline 3HHR-atomic & 3835.2 & 15976.8 & 223 & 28 & $5.5 \AA$ \\
\hline 3HHR-coarse & 3549.63 & 16345.7 & 67 & 28 & \\
\hline
\end{tabular}

TABLE 2

Direx versus Loopy: MSA for $n=500$ conformers. The number of residues comprises the two residues bounding all the conformers-these are common to all conformers.

\section{C.2 Geometric and Topological Assessment: Tables}

\begin{tabular}{|c|c|c|c|}
\hline PDB & $\frac{R_{G}}{R_{H}}$ & $\frac{n_{H_{100 \%}}}{n_{G}}$ & $\frac{n_{H_{95}}}{n_{G}}$ \\
\hline 1BTH-atomic & 1.11 & 112.67 & 6.0 \\
\hline 1CGI-atomic & 1.12 & 154.33 & 1.67 \\
\hline 1OAZ-atomic & 1.13 & 1.67 & 1.33 \\
\hline 3HHR-atomic & 1.09 & 9.67 & 2.67 \\
\hline \hline 1BTH-coarse & 1.14 & 67.2 & 3.6 \\
\hline 1CGI-coarse & 1.12 & 160.0 & 2.33 \\
\hline 1OAZ-coarse & 1.15 & 1.67 & 1.33 \\
\hline 3HHR-coarse & 1.11 & 10.67 & 2.67 \\
\hline
\end{tabular}

TABLE 3

\begin{tabular}{|c|c|c|c|}
\hline PDB & $\frac{R_{G}}{R_{H}}$ & $\frac{n_{H_{100 \%}}}{n_{G}}$ & $\frac{n_{H_{95}}}{n_{G}}$ \\
\hline 1BTH-atomic & 1.18 & 9.5 & 7.0 \\
\hline 1CGI-atomic & 1.14 & 79.5 & 28.17 \\
\hline 1OAZ-atomic & 1.21 & 42.64 & 10.82 \\
\hline 3HHR-atomic & 1.54 & 4.3 & 3.8 \\
\hline \hline 1BTH-coarse & 1.25 & 10.86 & 8.29 \\
\hline 1CGI-coarse & 1.34 & 79.5 & 11.0 \\
\hline 1OAZ-coarse & 1.27 & 44.67 & 10.89 \\
\hline 3HHR-coarse & 1.56 & 13.31 & 3.0 \\
\hline
\end{tabular}

TABLE 4

Direx data set. Comparison of the selection methods. Loopy data set. Comparison of the selection methods. See text for notations. See text for notations.

\begin{tabular}{|c||c|c|c|c|c||c|c|c|c|}
\hline PDB & $n_{G_{100}}$ & $m\left(\beta_{1}\right)$ & $M\left(\beta_{1}\right)$ & $\mu\left(\beta_{1}\right)$ & $\operatorname{med}\left(\beta_{1}\right)$ & $m\left(\beta_{1}\right)$ & $M\left(\beta_{1}\right)$ & $\mu\left(\beta_{1}\right)$ & $\operatorname{med}\left(\beta_{1}\right)$ \\
\hline 1BTH-atomic & 28 & 7 & 16 & 9.38 & 8 & 0 & 7 & 0.44 & 0 \\
\hline 1CGI-atomic & 10 & 2 & 12 & 8.06 & 8 & 0 & 8 & 0.45 & 0 \\
\hline 1OAZ-atomic & 13 & 5 & 21 & 7.07 & 6 & 0 & 19 & 0.44 & 0 \\
\hline 3HHR-atomic & 6 & 6 & 30 & 13.95 & 11.5 & 0 & 31 & 1.05 & 0 \\
\hline \hline 1BTH-coarse & 17 & 0 & 12 & 9.63 & 11 & 0 & 11 & 0.96 & 1 \\
\hline 1CGI-coarse & 22 & 0 & 17 & 6.49 & 7 & 0 & 8 & 1.14 & 1 \\
\hline 1OAZ-coarse & 14 & 1 & 18 & 9.79 & 10 & 0 & 14 & 1.53 & 2 \\
\hline 3HHR-coarse & 11 & 4 & 36 & 16.16 & 15 & 1 & 23 & 2.22 & 1 \\
\hline
\end{tabular}

TABLE 5

Direx data set. Comparing the evolution of the first Betti number up to $n_{G_{100 \%}}$ conformers selected; Left: Greedy; Right: HClust. $m, M, \mu$ and med respectively stand for min, max, mean, median.

\begin{tabular}{|c||c|c|c|c|c||c|c|c|c|}
\hline PDB & $n_{G_{100 \%}}$ & $m\left(\beta_{1}\right)$ & $M\left(\beta_{1}\right)$ & $\mu\left(\beta_{1}\right)$ & $\operatorname{med}\left(\beta_{1}\right)$ & $m\left(\beta_{1}\right)$ & $M\left(\beta_{1}\right)$ & $\mu\left(\beta_{1}\right)$ & $\operatorname{med}\left(\beta_{1}\right)$ \\
\hline 1BTH-atomic & 39 & 3 & 44 & 25.08 & 24 & 1 & 20 & 3.78 & 2 \\
\hline 1CGI-atomic & 16 & 4 & 38 & 23.68 & 22 & 1 & 27 & 2.6 & 2 \\
\hline 1OAZ-atomic & 37 & 4 & 43 & 25.82 & 24 & 4 & 18 & 7.58 & 5 \\
\hline 3HHR-atomic & 36 & 17 & 342 & 283.12 & 306 & 26 & 163 & 86.56 & 74 \\
\hline \hline 1BTH-coarse & 32 & 0 & 51 & 36.26 & 35 & 2 & 25 & 6.73 & 6 \\
\hline 1CGI-coarse & 21 & 0 & 59 & 30.15 & 26 & 0 & 30 & 5.67 & 5 \\
\hline 1OAZ-coarse & 28 & 0 & 77 & 61.67 & 63 & 7 & 35 & 19.99 & 19 \\
\hline 3HHR-coarse & 46 & 1 & 492 & 382.59 & 452 & 6 & 219 & 149.02 & 141 \\
\hline
\end{tabular}

TABLE 6

Loopy data set. Comparing the evolution of the first Betti number up to $n_{G_{100 \%}}$ conformers selected; Left: Greedy; Right: HClust. $m, M, \mu$ and med respectively stand for min, max, mean, median. 


\begin{tabular}{|c|c|c|c|c|c|c|c|}
\hline File & Loop generated by & Selected by & Selection size & Min. & Max. & $\delta$ & $\frac{\delta_{G}-\delta_{H}}{\delta_{H}}$ \\
\hline $1 \mathrm{BTH}$ & Loopy & Greedy & 10 & 2.767 & 10.15 & 7.383 & 0.392 \\
\hline $1 \mathrm{BTH}$ & Loopy & HClust & 10 & 2.693 & 7.996 & 5.303 & \\
\hline 1BTH & Loopy & Greedy & 15 & 2.767 & 10.15 & 7.383 & 0.315 \\
\hline $1 \mathrm{BTH}$ & Loopy & HClust & 15 & 2.693 & 8.307 & 5.614 & \\
\hline 1BTH & Loopy & Greedy & 30 & 2.581 & 10.15 & 7.569 & -0.052 \\
\hline $1 \mathrm{BTH}$ & Loopy & HClust & 30 & 2.162 & 10.15 & 7.988 & \\
\hline "1BTH & $\overline{\text { Direx }}$ & $\overline{~ G r e e d y ~}$ & $\overline{\overline{10}}$ & 0.5627 & 3.501 & 2.9383 & 0.184 \\
\hline $1 \mathrm{BTH}$ & Direx & HClust & 10 & 0.9152 & 3.396 & 2.4808 & \\
\hline 1BTH & Direx & Greedy & 15 & 0.5627 & 3.501 & 2.9383 & 0.184 \\
\hline $1 \mathrm{BTH}$ & Direx & HClust & 15 & 0.9152 & 3.396 & 2.4808 & \\
\hline 1BTH & Direx & Greedy & 30 & 0.5627 & 3.679 & 3.1163 & 0.203 \\
\hline $1 \mathrm{BTH}$ & Direx & HClust & 30 & 0.8562 & 3.446 & 2.5898 & \\
\hline 1CGI & Loopy & Greedy & 10 & 3.614 & 10.4 & 6.786 & 0.27 \\
\hline 1CGI & Loopy & HClust & 10 & 3.413 & 8.755 & 5.342 & \\
\hline 1CGI & Loopy & Greedy & 15 & 3.614 & 10.91 & 7.296 & 0.326 \\
\hline 1CGI & Loopy & HClust & 15 & 3.413 & 8.916 & 5.503 & \\
\hline 1CGI & Loopy & Greedy & 30 & 3.614 & 10.95 & 7.336 & -0.078 \\
\hline 1CGI & Loopy & HClust & 30 & 2.441 & 10.4 & 7.959 & \\
\hline $\begin{array}{l}1 C G I \\
\end{array}$ & Direx & Greedy & 10 & 0.7777 & $\bar{~} 4.442$ & 3.6643 & 0.637 \\
\hline 1CGI & Direx & HClust & 10 & 1.364 & 3.602 & 2.238 & \\
\hline 1CGI & Direx & Greedy & 15 & 0.7777 & 4.442 & 3.6643 & 0.336 \\
\hline 1CGI & Direx & HClust & 15 & 1.364 & 4.106 & 2.742 & \\
\hline 1CGI & Direx & Greedy & 30 & 0.7777 & 4.442 & 3.6643 & 0.336 \\
\hline 1CGI & Direx & HClust & 30 & 1.364 & 4.106 & 2.742 & \\
\hline 1OAZ & Loopy & Greedy & 10 & $\overline{4.237}$ & 17.32 & 13.083 & $\overline{0.58}$ \\
\hline 1OAZ & Loopy & HClust & 10 & 3.327 & 11.61 & 8.283 & \\
\hline 1OAZ & Loopy & Greedy & 15 & 4.237 & 17.35 & 13.113 & 0.392 \\
\hline 1OAZ & Loopy & HClust & 15 & 3.079 & 12.5 & 9.421 & \\
\hline 1OAZ & Loopy & Greedy & 30 & 2.716 & 17.35 & 14.634 & 0.68 \\
\hline 1OAZ & Loopy & HClust & 30 & 3.079 & 11.79 & 8.711 & \\
\hline 1OAZ & Direx & Greedy & 10 & 0.8034 & 6.967 & 6.1636 & 0.353 \\
\hline 1OAZ & Direx & HClust & 10 & 1.933 & 6.49 & 4.557 & \\
\hline 1OAZ & Direx & Greedy & 15 & 0.8034 & 6.967 & 6.1636 & 0.219 \\
\hline 1OAZ & Direx & HClust & 15 & 1.111 & 6.169 & 5.058 & \\
\hline 1OAZ & Direx & Greedy & 30 & 0.8034 & 6.967 & 6.1636 & 0.221 \\
\hline 1OAZ & Direx & HClust & 30 & 1.111 & 6.159 & 5.048 & \\
\hline 3HHR & $\overline{\text { Loopy }}$ & Greedy & $\overline{10}$ & $\begin{array}{c}8.517 \\
\end{array}$ & 21.16 & 12.643 & -0.288 \\
\hline $3 \mathrm{HHR}$ & Loopy & HClust & 10 & 5.521 & 23.27 & 17.749 & \\
\hline $3 \mathrm{HHR}$ & Loopy & Greedy & 15 & 8.517 & 23.27 & 14.753 & -0.169 \\
\hline $3 \mathrm{HHR}$ & Loopy & HClust & 15 & 5.521 & 23.27 & 17.749 & \\
\hline 3HHR & Loopy & Greedy & 30 & 8.517 & 23.27 & 14.753 & -0.169 \\
\hline $3 \mathrm{HHR}$ & Loopy & HClust & 30 & 5.521 & 23.27 & 17.749 & \\
\hline 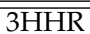 & 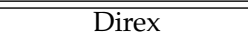 & 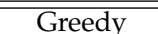 & $\overline{\overline{10}}$ & 0.4793 & 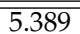 & 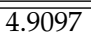 & 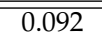 \\
\hline $3 \mathrm{HHR}$ & Direx & HClust & 10 & 0.8399 & 5.335 & 4.4951 & \\
\hline $3 \mathrm{HHR}$ & Direx & Greedy & 15 & 0.4793 & 5.485 & 5.0057 & 0.114 \\
\hline $3 \mathrm{HHR}$ & Direx & HClust & 15 & 0.8399 & 5.335 & 4.4951 & \\
\hline 3HHR & Direx & Greedy & 30 & 0.4793 & 5.485 & 5.0057 & 0.111 \\
\hline $3 \mathrm{HHR}$ & Direx & HClust & 30 & 0.8399 & 5.346 & 4.5061 & \\
\hline
\end{tabular}

TABLE 7

Selection by HClust versus selection by Greedy for coarse models: comparison of $C_{\alpha}$-rmsd of loops selected with respect to the native bound loop. $\delta=\operatorname{Max}-\operatorname{Min}$, the subscript $G$ or $H$ standing for the algorithm used, be it 


\begin{tabular}{|c|c|c|c|c|c|c|c|}
\hline File & Loop generated by & Selected by & Selection size & Min. & Max. & $\delta$ & $\frac{\delta_{G}-\delta_{H}}{\delta_{H}}$ \\
\hline $1 \mathrm{BTH}$ & Loopy & Greedy & 10 & 2.693 & 10.15 & 7.457 & 0.406 \\
\hline $1 \mathrm{BTH}$ & Loopy & HClust & 10 & 2.693 & 7.996 & 5.303 & \\
\hline 1BTH & Loopy & Greedy & 15 & 2.581 & 10.15 & 7.569 & 0.348 \\
\hline $1 \mathrm{BTH}$ & Loopy & HClust & 15 & 2.693 & 8.307 & 5.614 & \\
\hline 1BTH & Loopy & Greedy & 30 & 2.581 & 10.15 & 7.569 & -0.052 \\
\hline $1 \mathrm{BTH}$ & Loopy & HClust & 30 & 2.162 & 10.15 & 7.988 & \\
\hline "1BTH & $\overline{\text { Direx }}$ & $\overline{~ G r e e d y ~}$ & $\overline{\overline{10}}$ & 0.9006 & $\bar{~} 3.528$ & 2.6274 & $\overline{0.059}$ \\
\hline $1 \mathrm{BTH}$ & Direx & HClust & 10 & 0.9152 & 3.396 & 2.4808 & \\
\hline 1BTH & Direx & Greedy & 15 & 0.9006 & 3.679 & 2.7784 & 0.12 \\
\hline $1 \mathrm{BTH}$ & Direx & HClust & 15 & 0.9152 & 3.396 & 2.4808 & \\
\hline 1BTH & Direx & Greedy & 30 & 0.9006 & 3.679 & 2.7784 & 0.073 \\
\hline $1 \mathrm{BTH}$ & Direx & HClust & 30 & 0.8562 & 3.446 & 2.5898 & \\
\hline 1CGI & Loopy & Greedy & $\overline{10}$ & 5.934 & 10.55 & 4.616 & -0.136 \\
\hline 1CGI & Loopy & HClust & 10 & 3.413 & 8.755 & 5.342 & \\
\hline 1CGI & Loopy & Greedy & 15 & 3.614 & 10.91 & 7.296 & 0.326 \\
\hline 1CGI & Loopy & HClust & 15 & 3.413 & 8.916 & 5.503 & \\
\hline 1CGI & Loopy & Greedy & 30 & 3.614 & 10.95 & 7.336 & $\begin{array}{c}-0.078 \\
\end{array}$ \\
\hline 1CGI & Loopy & HClust & 30 & 2.441 & 10.4 & 7.959 & \\
\hline "1CGI & $\overline{\text { Direx }}$ & Greedy & $\overline{10}$ & 1.45 & 4.442 & 2.992 & 0.337 \\
\hline 1CGI & Direx & HClust & 10 & 1.364 & 3.602 & 2.238 & \\
\hline 1CGI & Direx & Greedy & 15 & 1.45 & 4.442 & 2.992 & 0.091 \\
\hline 1CGI & Direx & HClust & 15 & 1.364 & 4.106 & 2.742 & \\
\hline 1CGI & Direx & Greedy & 30 & 1.437 & 4.442 & 3.005 & 0.096 \\
\hline 1CGI & Direx & HClust & 30 & 1.364 & 4.106 & 2.742 & \\
\hline 10AZ & Loopy & Greedy & $\overline{10}$ & 2.716 & 17.35 & 14.634 & 0.767 \\
\hline 1OAZ & Loopy & HClust & 10 & 3.327 & 11.61 & 8.283 & \\
\hline 1OAZ & Loopy & Greedy & 15 & 2.716 & 17.35 & 14.634 & 0.553 \\
\hline 1OAZ & Loopy & HClust & 15 & 3.079 & 12.5 & 9.421 & \\
\hline 1OAZ & Loopy & Greedy & 30 & 2.716 & 17.35 & 14.634 & 0.68 \\
\hline 1OAZ & Loopy & HClust & 30 & 3.079 & 11.79 & 8.711 & \\
\hline 1OAZ & Direx & Greedy & 10 & 0.8034 & 6.71 & 5.9066 & 0.296 \\
\hline 1OAZ & Direx & HClust & 10 & 1.933 & 6.49 & 4.557 & \\
\hline 1OAZ & Direx & Greedy & 15 & 0.8034 & 6.71 & 5.9066 & 0.168 \\
\hline 1OAZ & Direx & HClust & 15 & 1.111 & 6.169 & 5.058 & \\
\hline 1OAZ & Direx & Greedy & 30 & 0.8034 & 6.71 & 5.9066 & 0.17 \\
\hline 1OAZ & Direx & HClust & 30 & 1.111 & 6.159 & 5.048 & \\
\hline /3HHR & Loopy & Greedy & $\overline{10}$ & $\begin{array}{l}11.11 \\
\end{array}$ & 23.27 & 12.16 & -0.315 \\
\hline $3 \mathrm{HHR}$ & Loopy & HClust & 10 & 5.521 & 23.27 & 17.749 & \\
\hline $3 \mathrm{HHR}$ & Loopy & Greedy & 15 & 11.11 & 23.27 & 12.16 & -0.315 \\
\hline $3 \mathrm{HHR}$ & Loopy & HClust & 15 & 5.521 & 23.27 & 17.749 & \\
\hline 3HHR & Loopy & Greedy & 30 & 8.611 & 23.27 & 14.659 & -0.174 \\
\hline $3 \mathrm{HHR}$ & Loopy & HClust & 30 & 5.521 & 23.27 & 17.749 & \\
\hline $\bar{~} \overline{3 \mathrm{HHR}}$ & 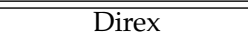 & 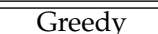 & $\overline{\overline{10}}$ & $\bar{~} 1.37$ & 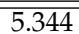 & $=3.974$ & "-0.116 \\
\hline $3 \mathrm{HHR}$ & Direx & HClust & 10 & 0.8399 & 5.335 & 4.4951 & \\
\hline 3HHR & Direx & Greedy & 15 & 1.37 & 5.344 & 3.974 & -0.116 \\
\hline $3 \mathrm{HHR}$ & Direx & HClust & 15 & 0.8399 & 5.335 & 4.4951 & \\
\hline 3HHR & Direx & Greedy & 30 & 1.37 & 5.344 & 3.974 & -0.118 \\
\hline $3 \mathrm{HHR}$ & Direx & HClust & 30 & 0.8399 & 5.346 & 4.5061 & \\
\hline
\end{tabular}

TABLE 8

Selection by HClust versus selection by Greedy for atomic models: comparison of $C_{\alpha}$-rmsd of loops selected with respect to the native bound loop. $\delta=\operatorname{Max}-\operatorname{Min}$, the subscript $G$ or $H$ standing for the algorithm used, be it

Greedy or HClust.

\section{C.3 Geometric and Topological Assessment: Graphs}

This section presents the plots of the variation of the MSA and of the Betti number $\beta_{1}$, as a function of the selection size. For each statistic, each model features four plots: $\{$ Direx, Loopy $\} \times\{$ atomic resolution, coarse grain $\}$. 


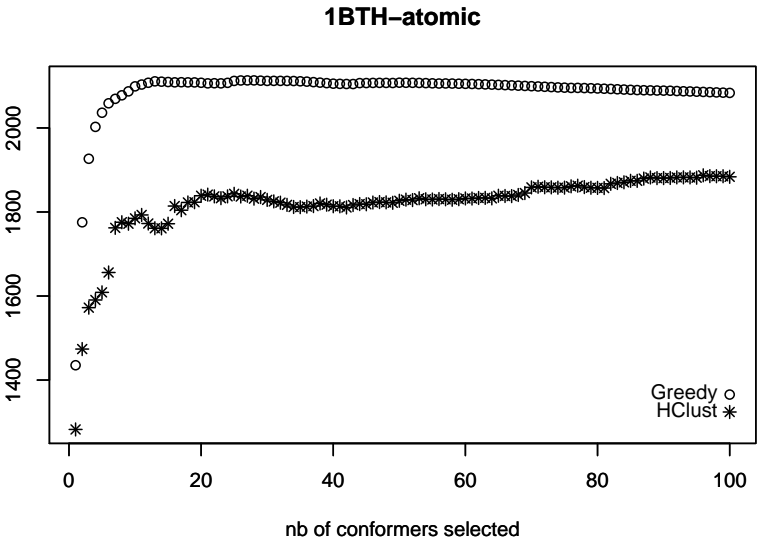

Direx

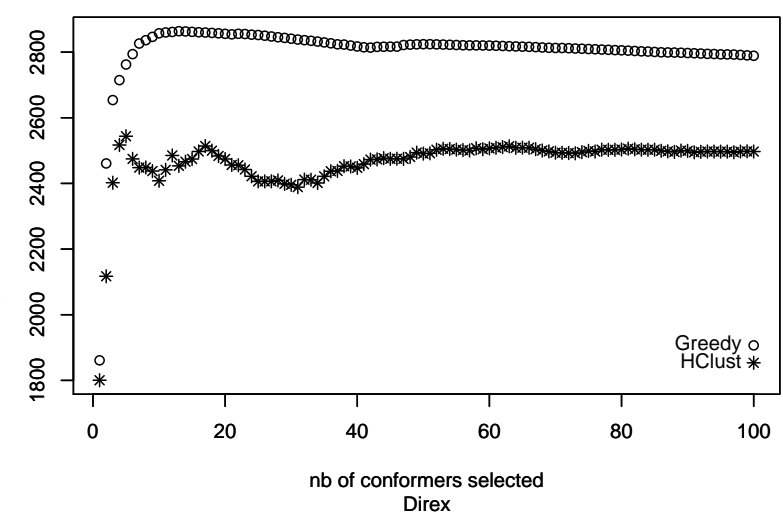

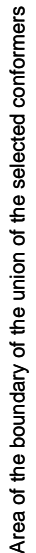

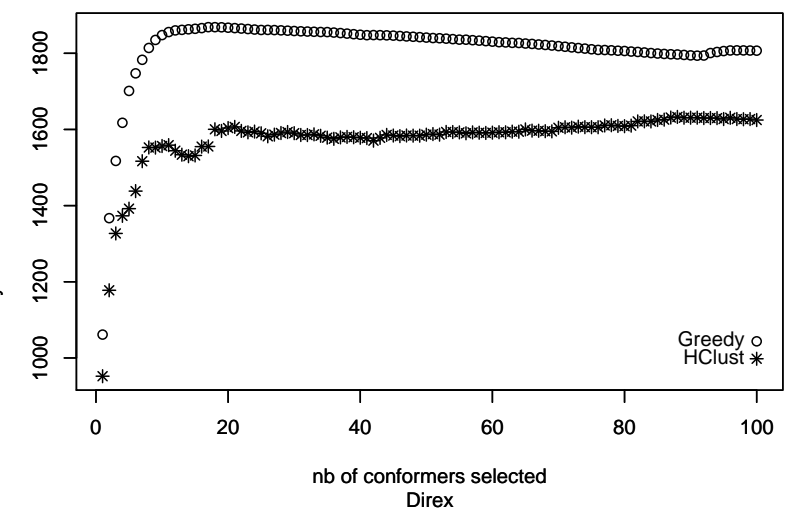

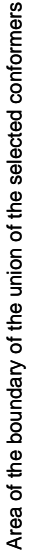

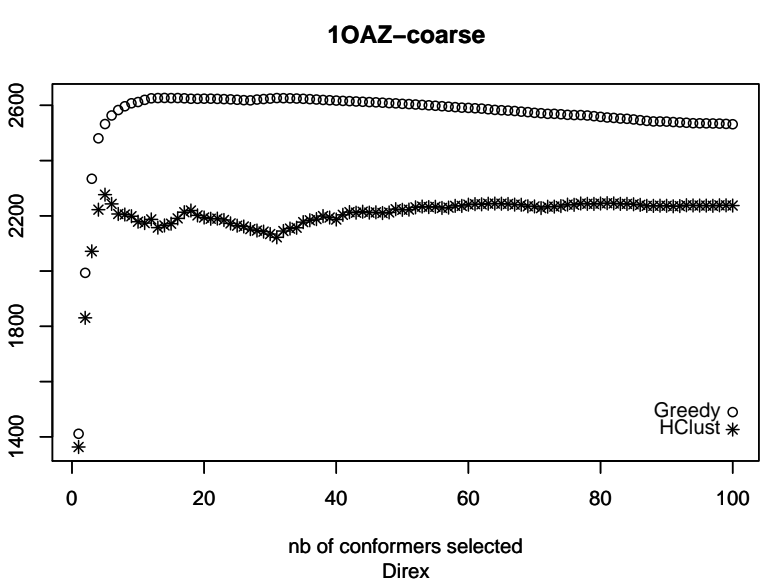

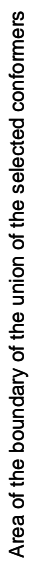

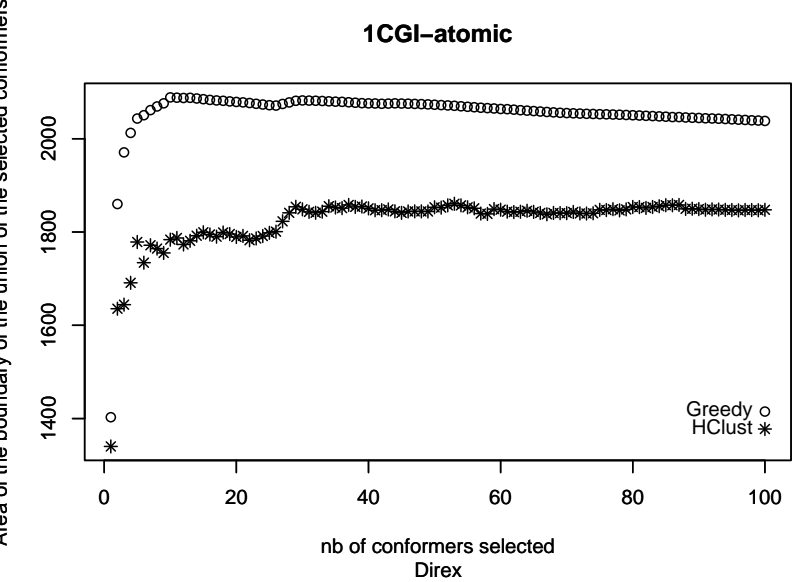

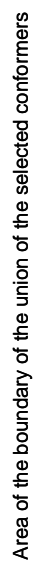

Direx
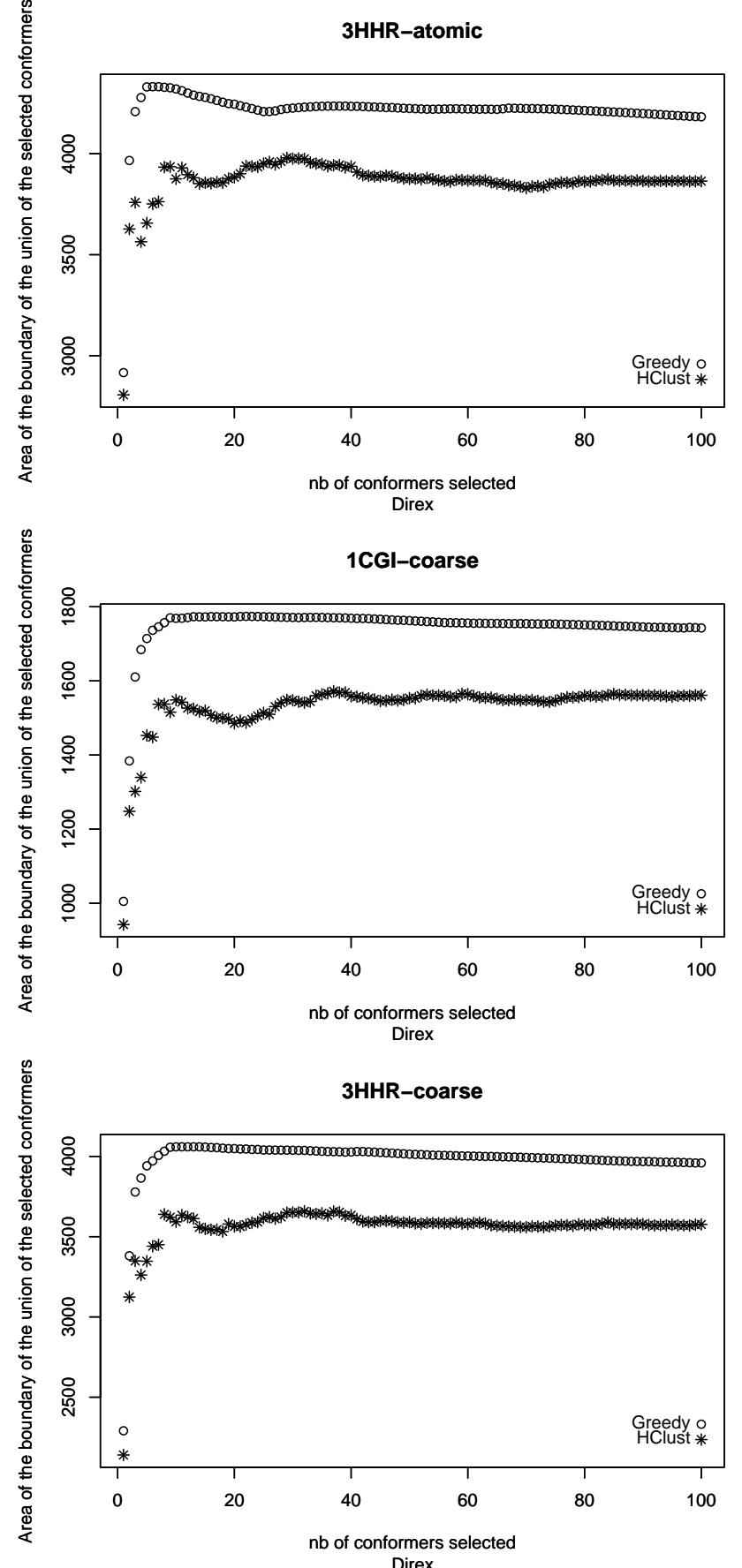

Direx 


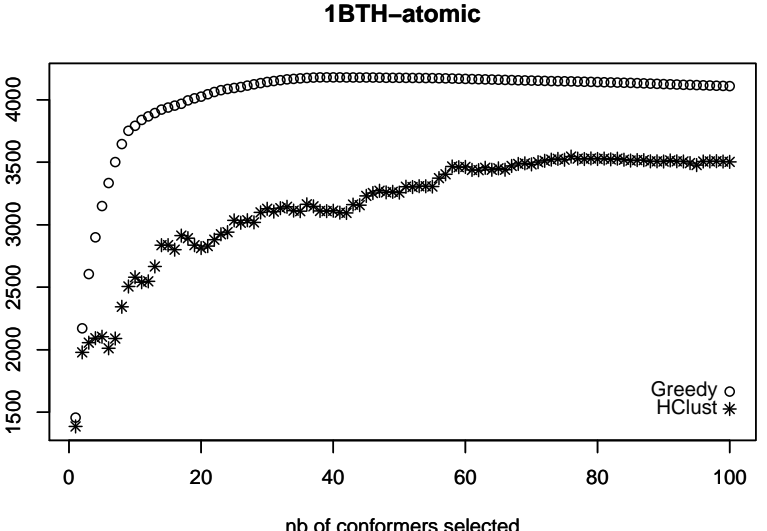

Loopy

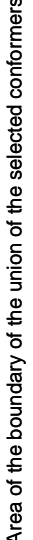

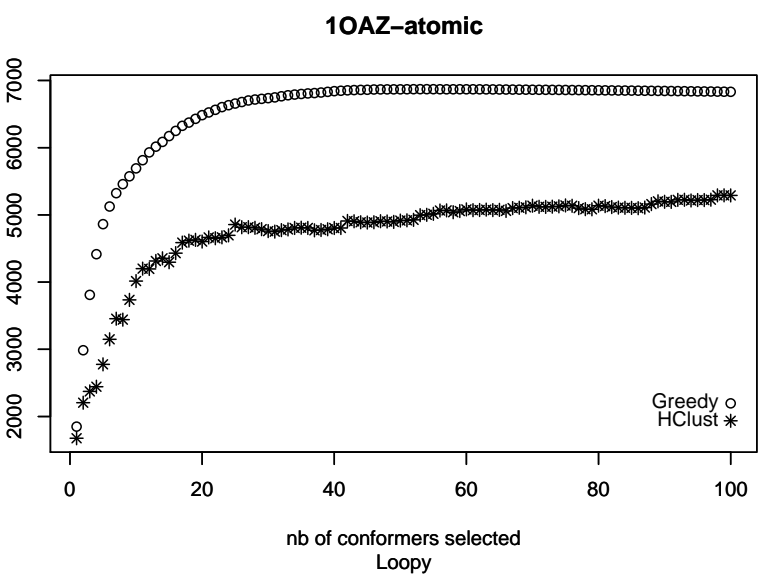

1BTH-coarse

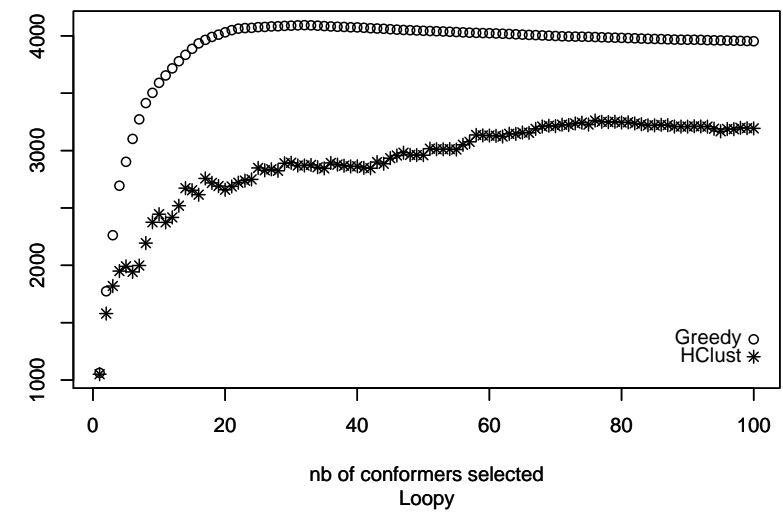

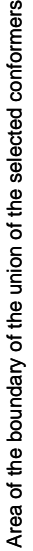

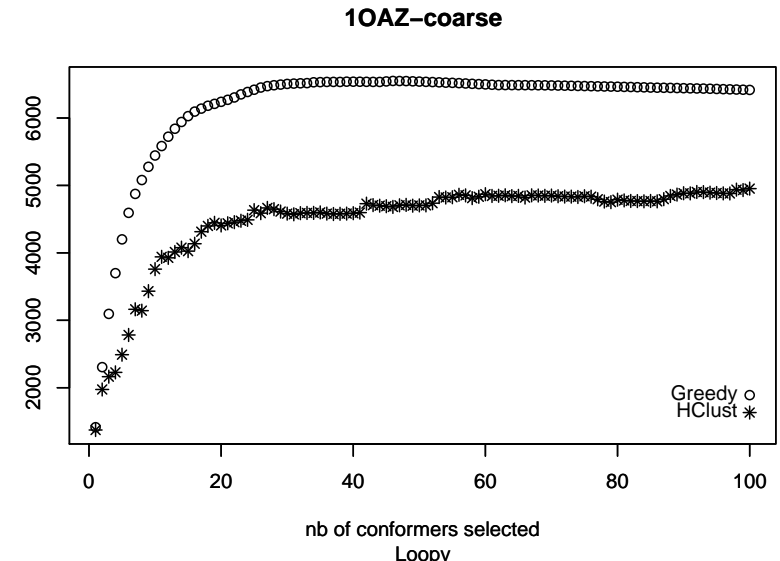

1CGI-atomic

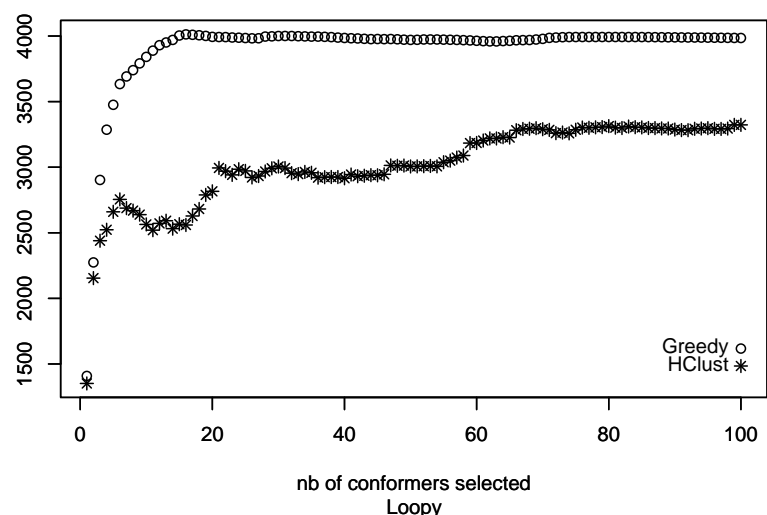

3HHR-atomic
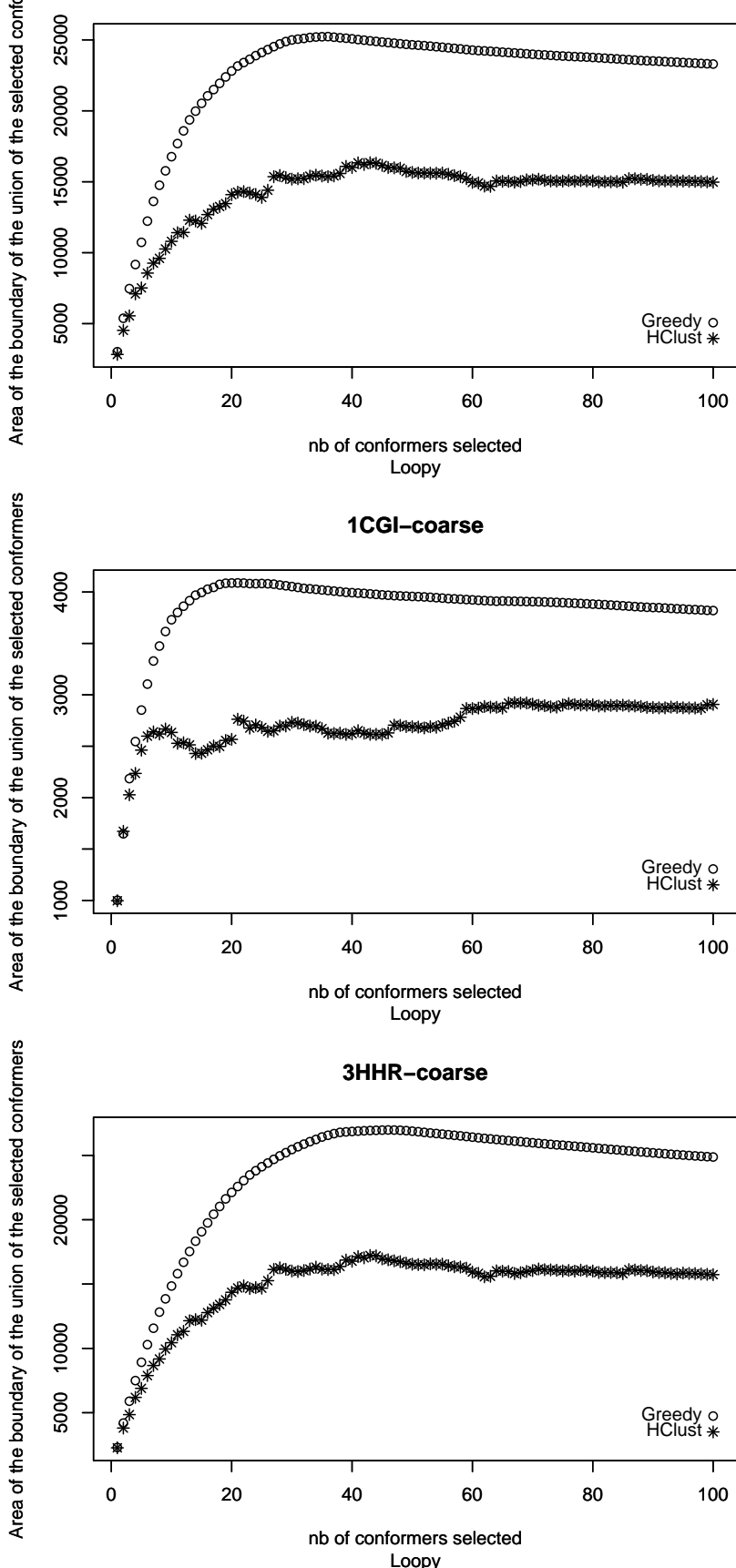

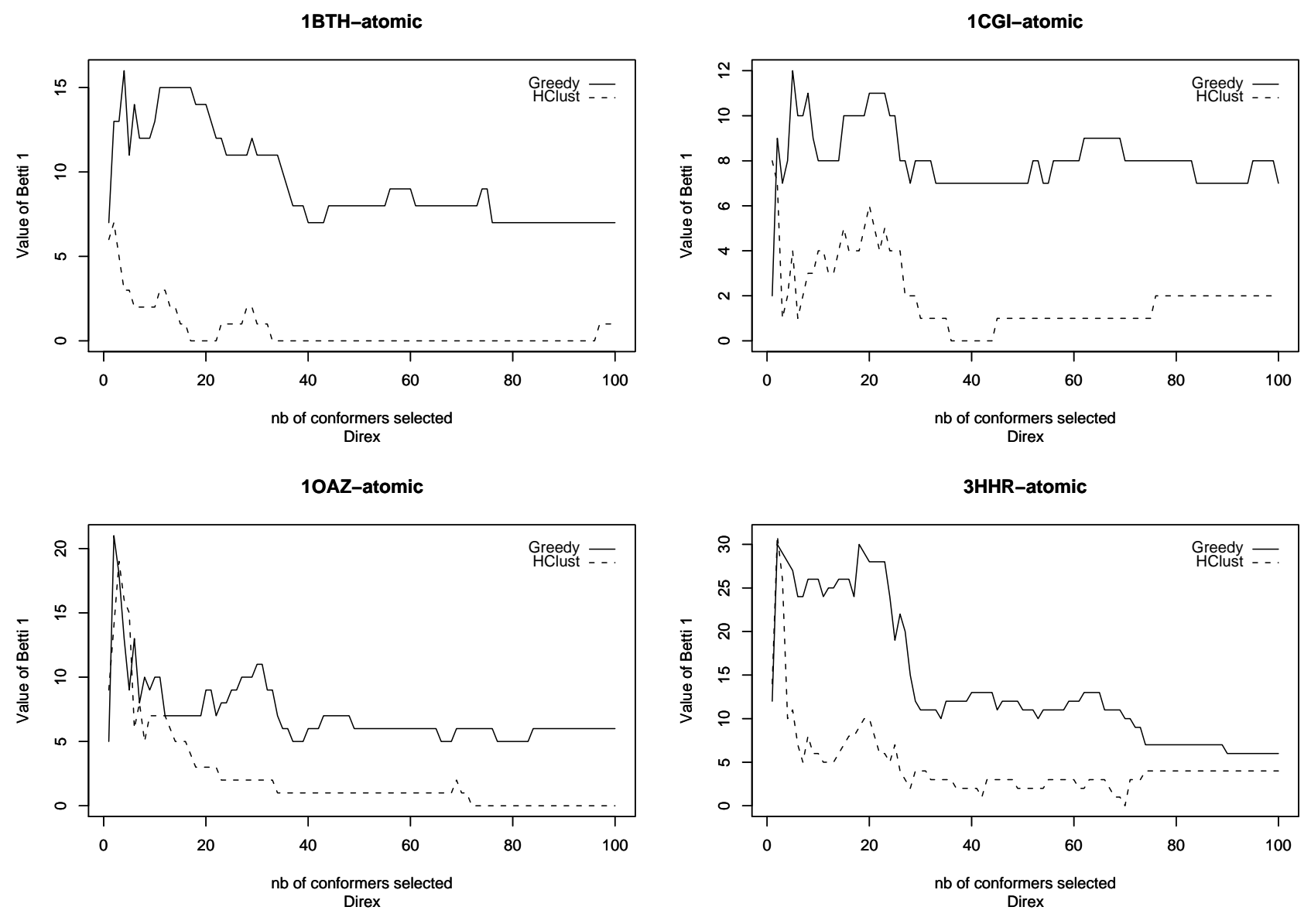

1BTH-coarse
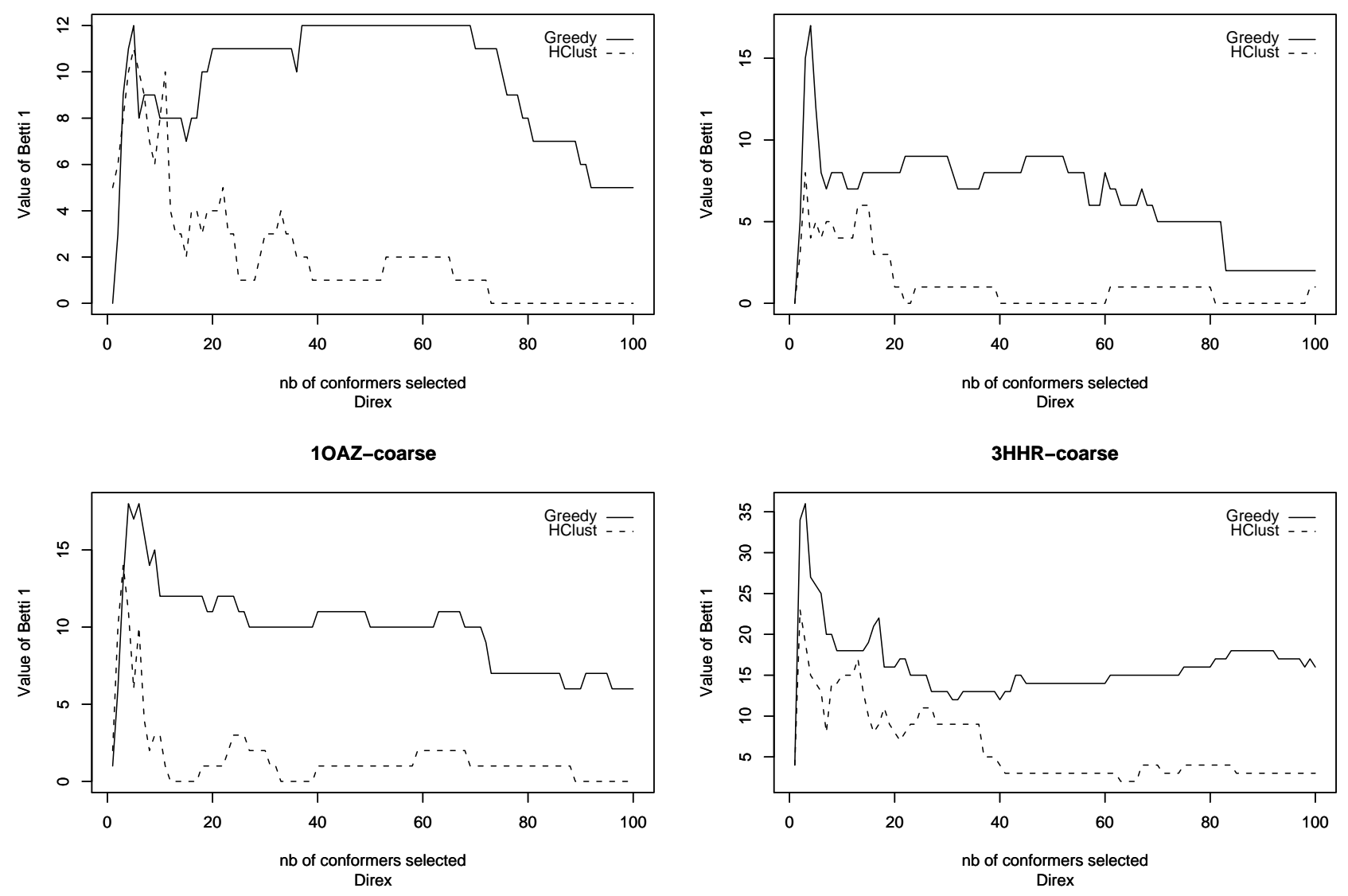

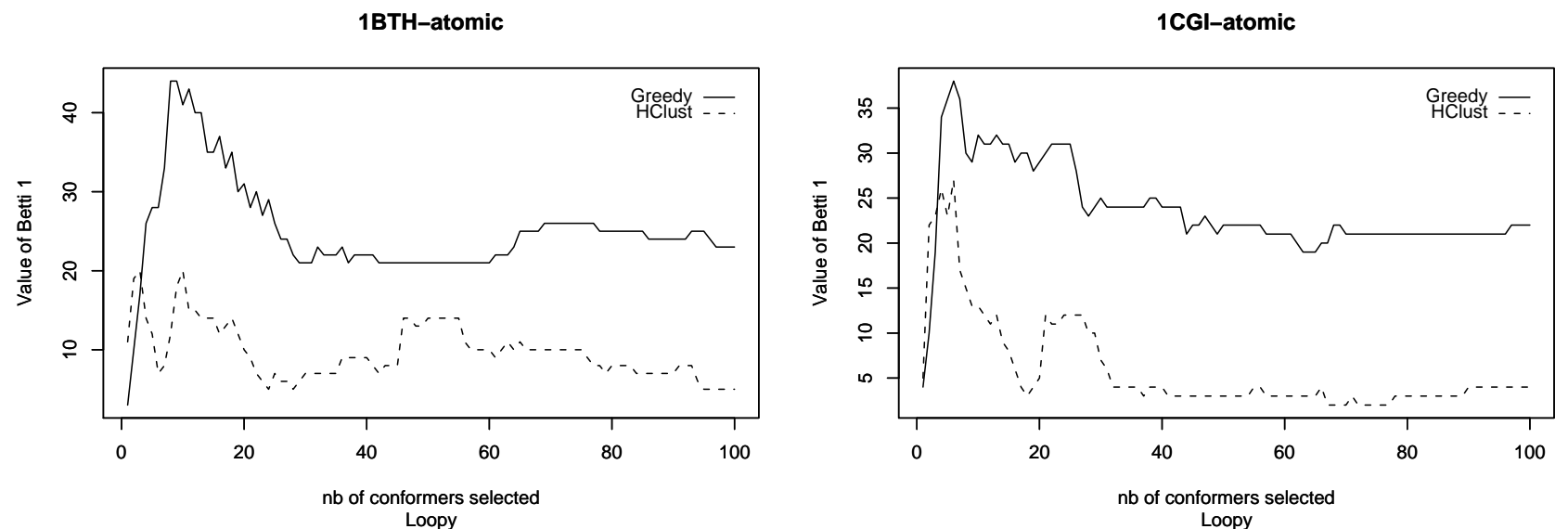

Loopy
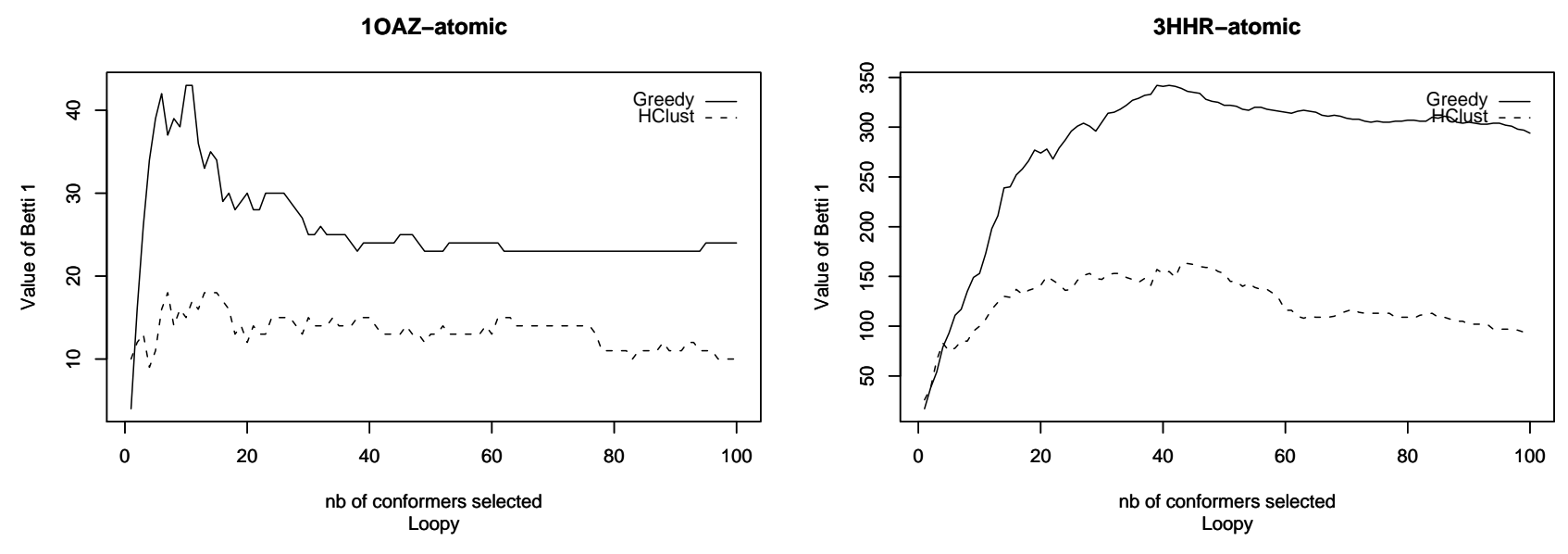

1BTH-coarse
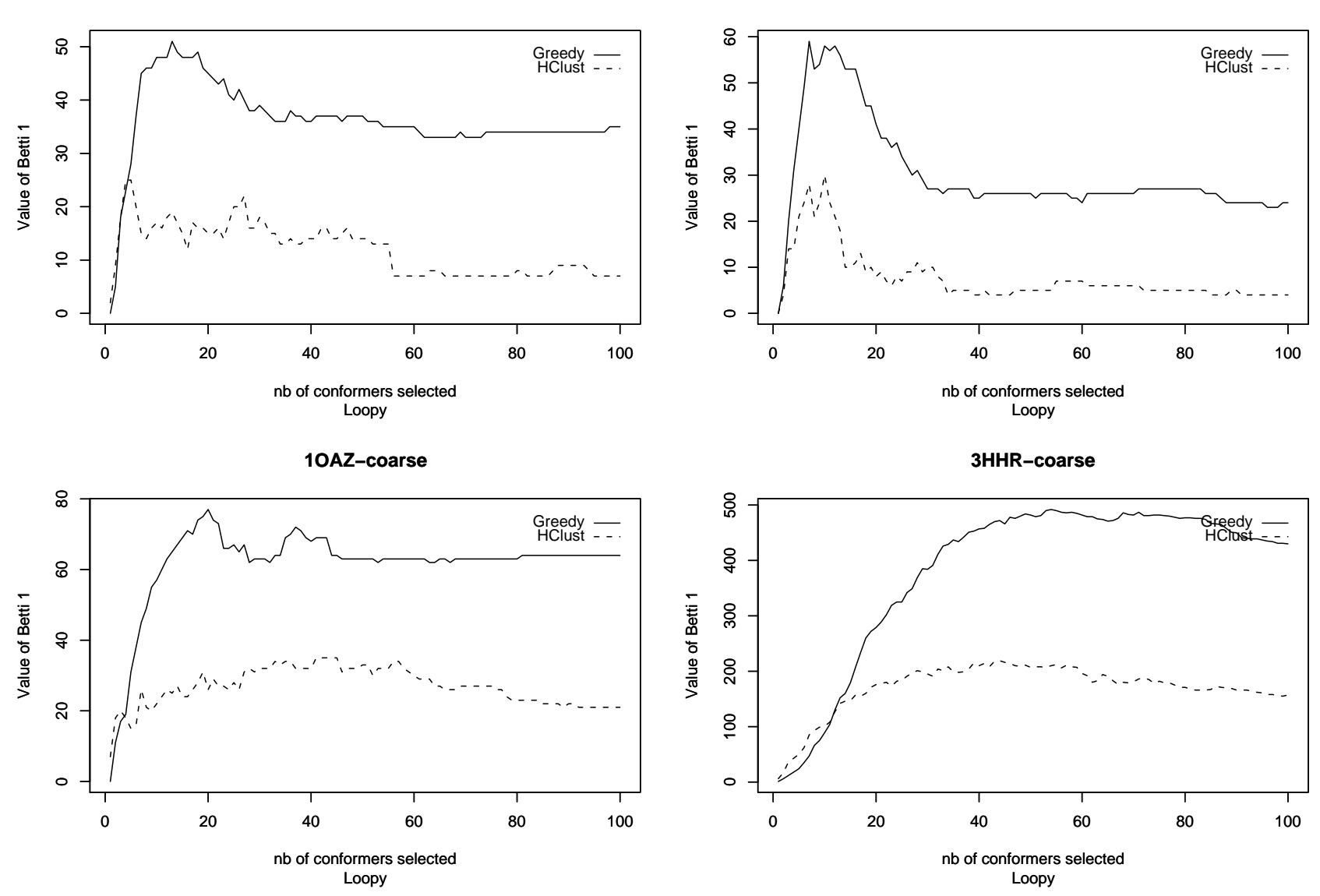


\section{C.4 Graphs: Docking Assessment}

For a given system, the 6 plots are organized as follows: the first two correspond to the sanity check B/B/B1 and U/B/B-1; the next (respectively last) two, namely B/B/B-HClust-10 and B/B/B-Greedy-10 (respectively U/B/B-HClust-10, U/B/B-Greedy-10) allow the comparison of Greedy and HClust for the Bound (respectively Unbound) version of the receptor. Recall that flexible regions on $1 \mathrm{BTH}, 1 \mathrm{CGI}$ and $1 \mathrm{OAZ}$ feature 10, 11 and 12 amino acids respectively on their receptor. 
1BTH B/B/B-1

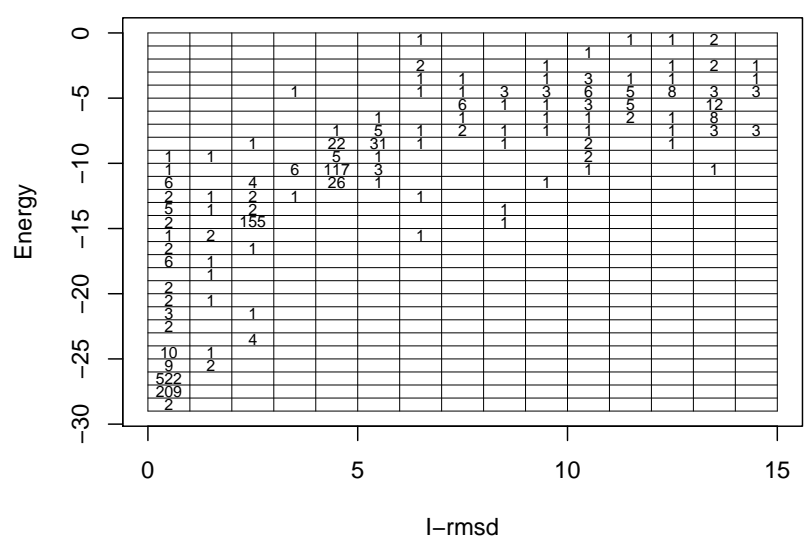

1BTH U/B/B-1

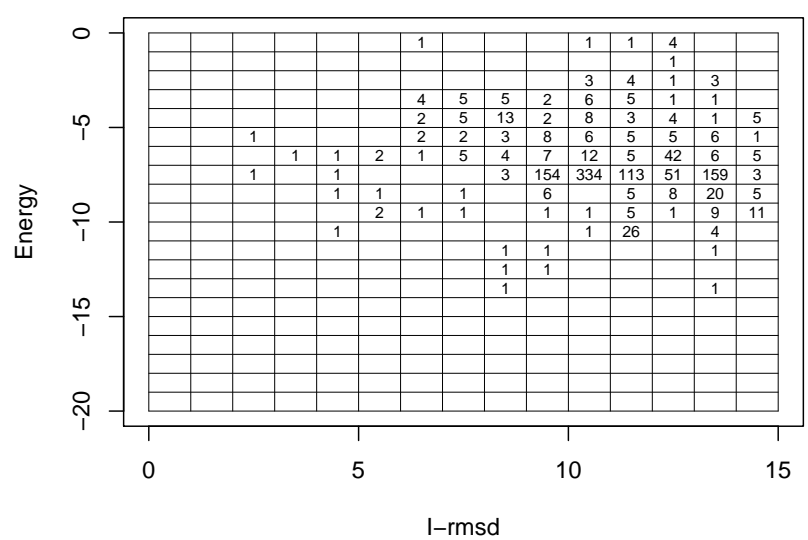

Fig. 12. 1BтH: binning the docking tests using the Bound and Unbound forms of the receptor. See text for details.
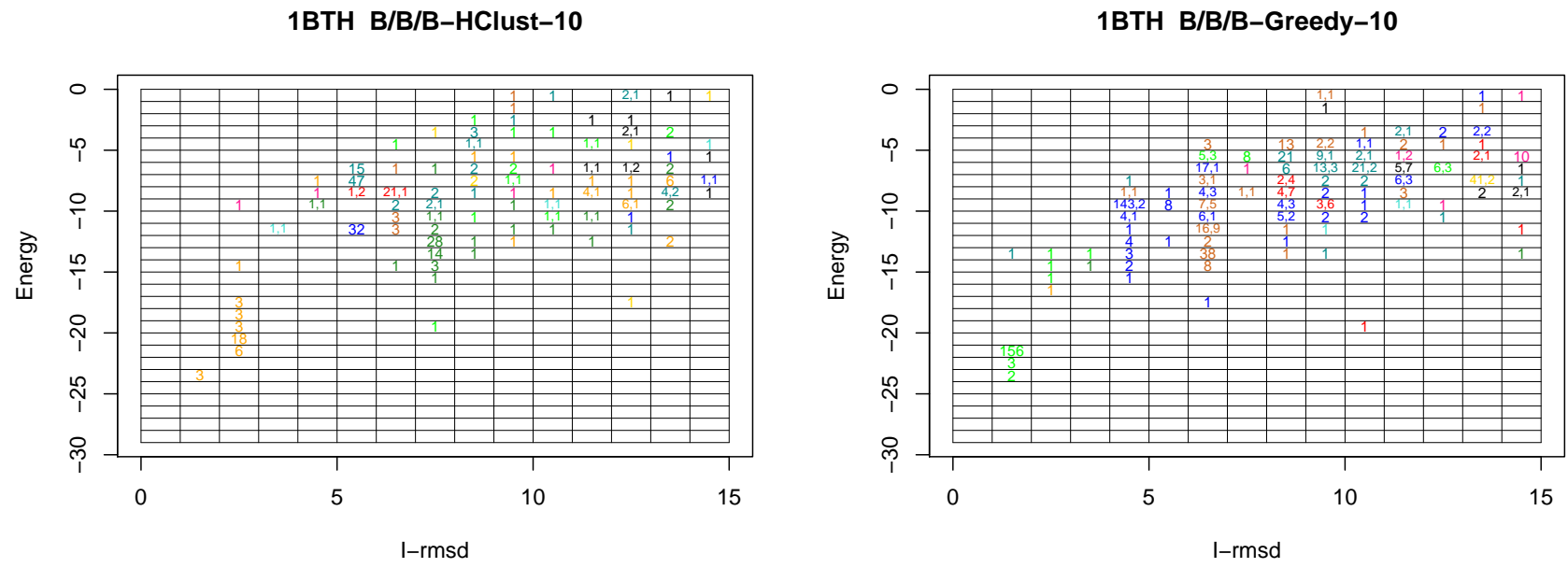

Fig. 13. 1BTH: binning the docking tests using the Bound form of the receptor. See text for details.
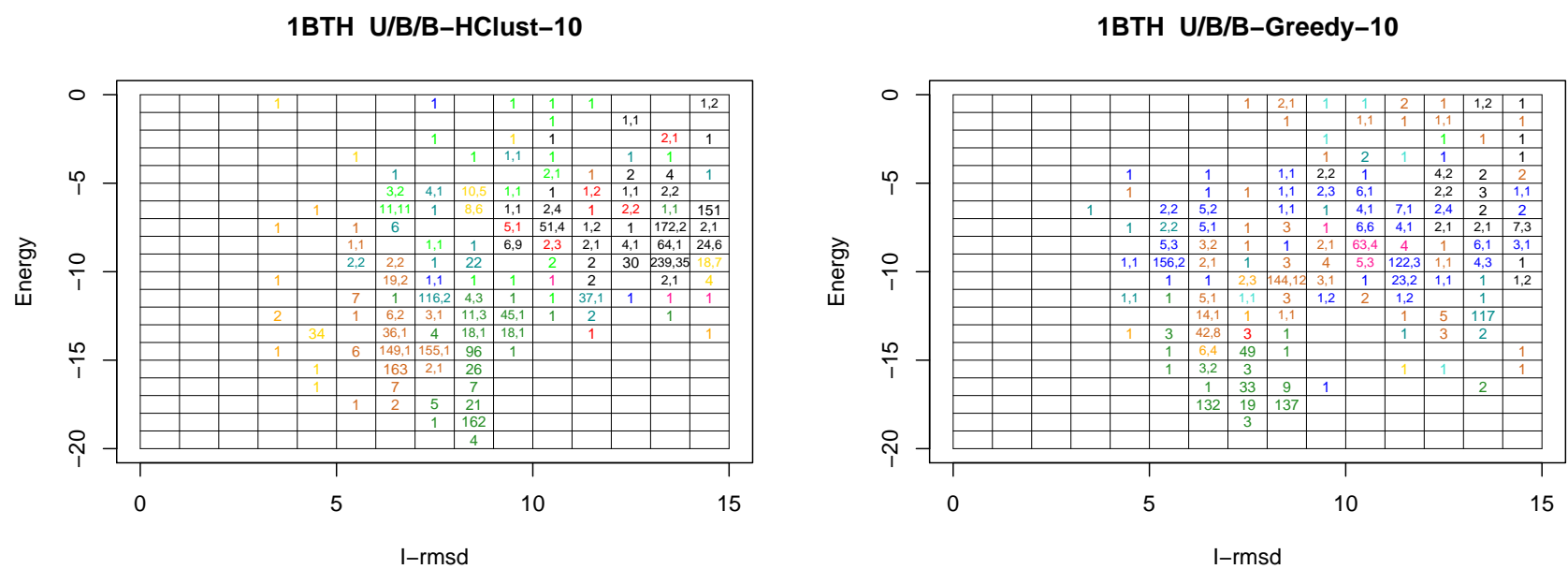

Fig. 14. 1BTH: binning the docking tests using the Unbound form of the receptor. See text for details. 
1CGI B/B/B-1

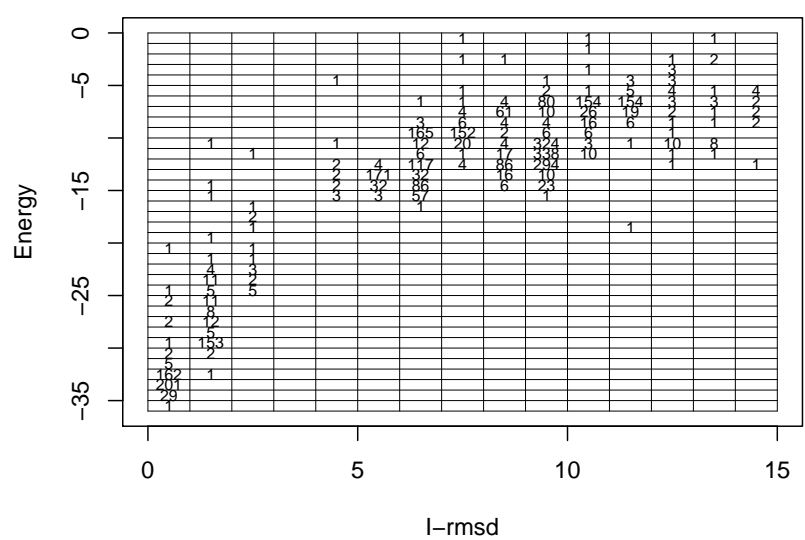

1CGI U/B/B-1

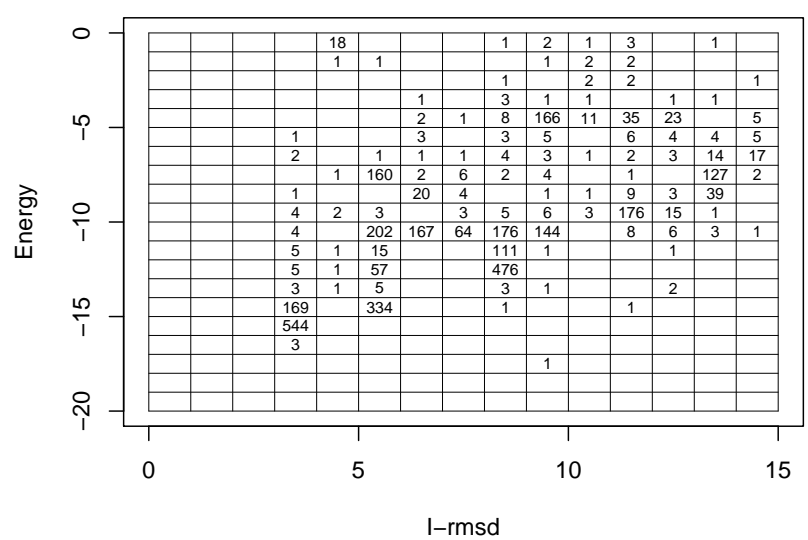

Fig. 15. 1CGI: binning the docking tests using the Bound and Unbound forms of the receptor. See text for details.

1CGI B/B/B-HClust-10

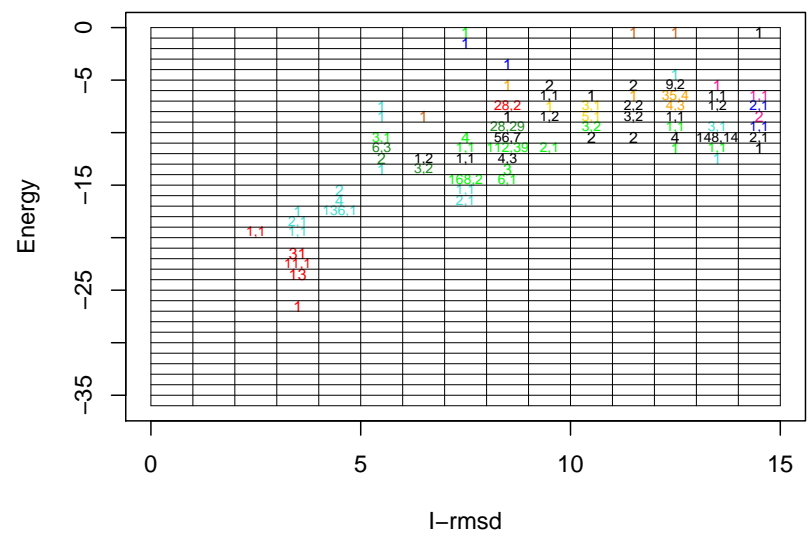

1CGI B/B/B-Greedy-10

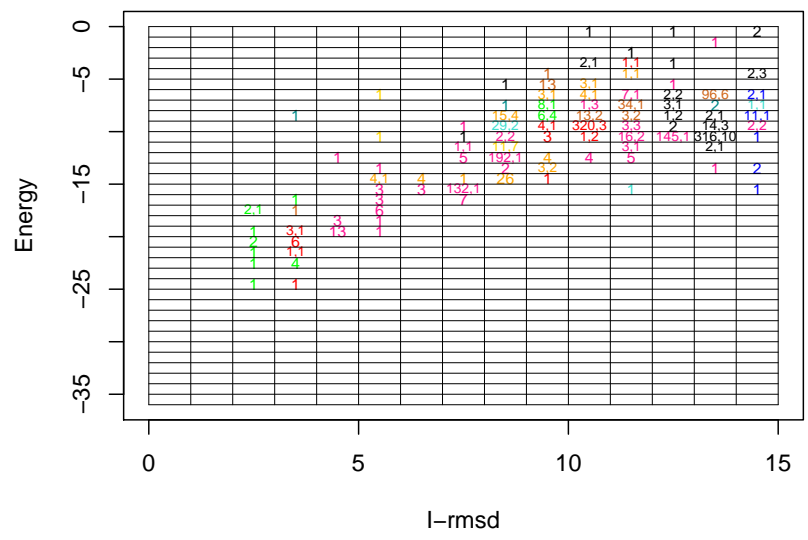

Fig. 16. 1CGI: binning the docking tests using the Bound form of the receptor. See text for details.
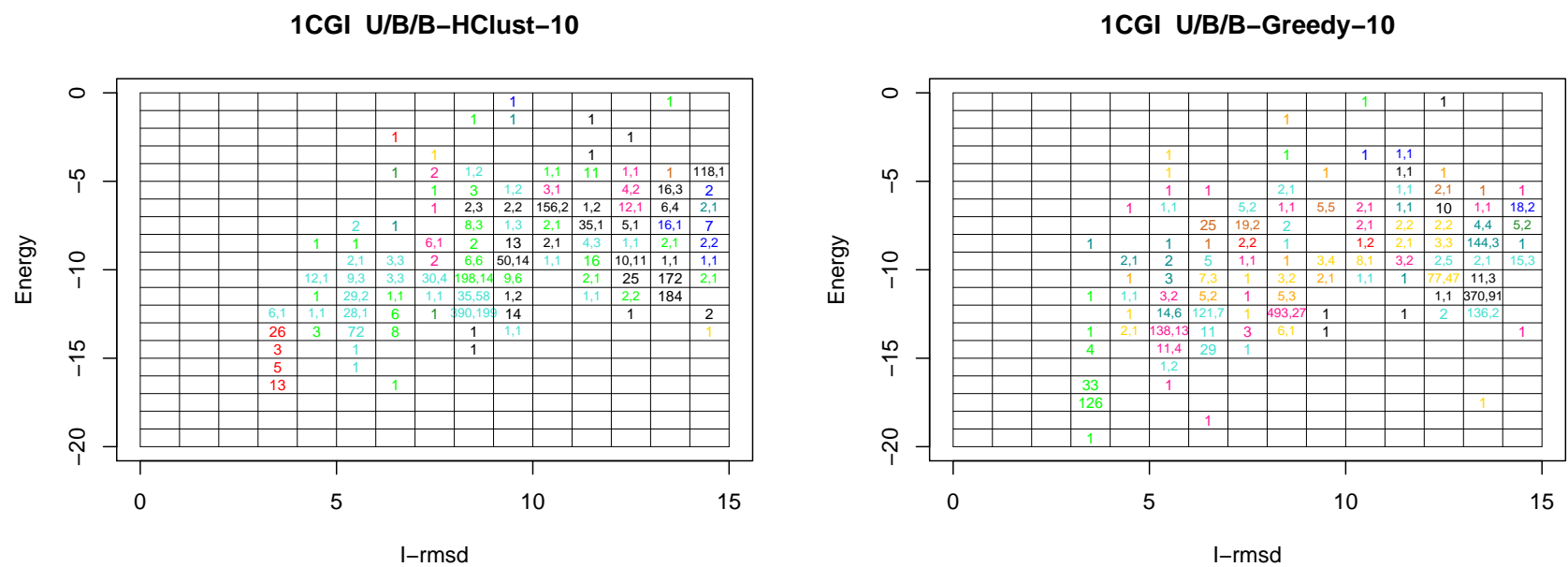

Fig. 17. 1CGI: binning the docking tests using the Unbound form of the receptor. See text for details. 
1OAZ B/B/B-1

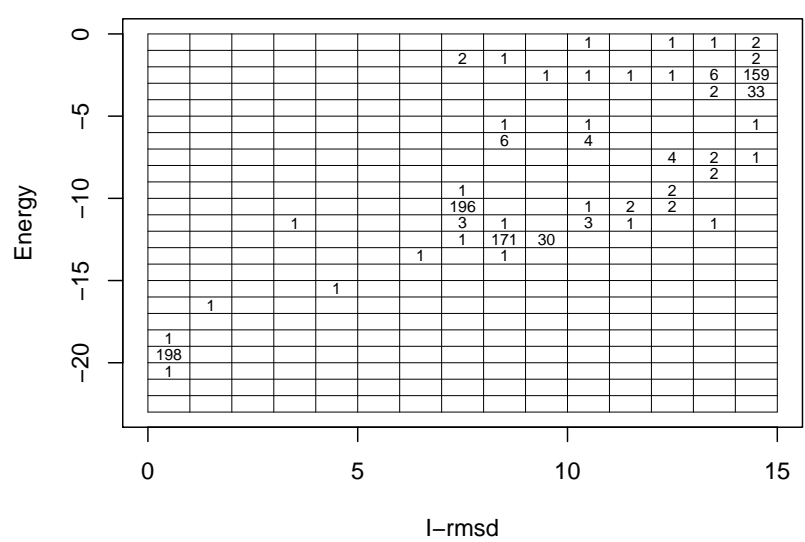

$10 A Z$ U/B/B-1

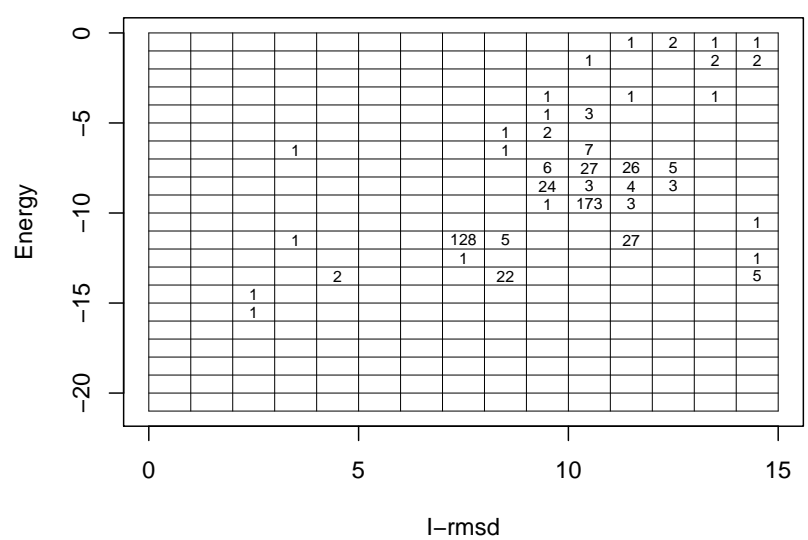

Fig. 18. 10Az: binning the docking tests using the Bound and Unbound forms of the receptor. See text for details.
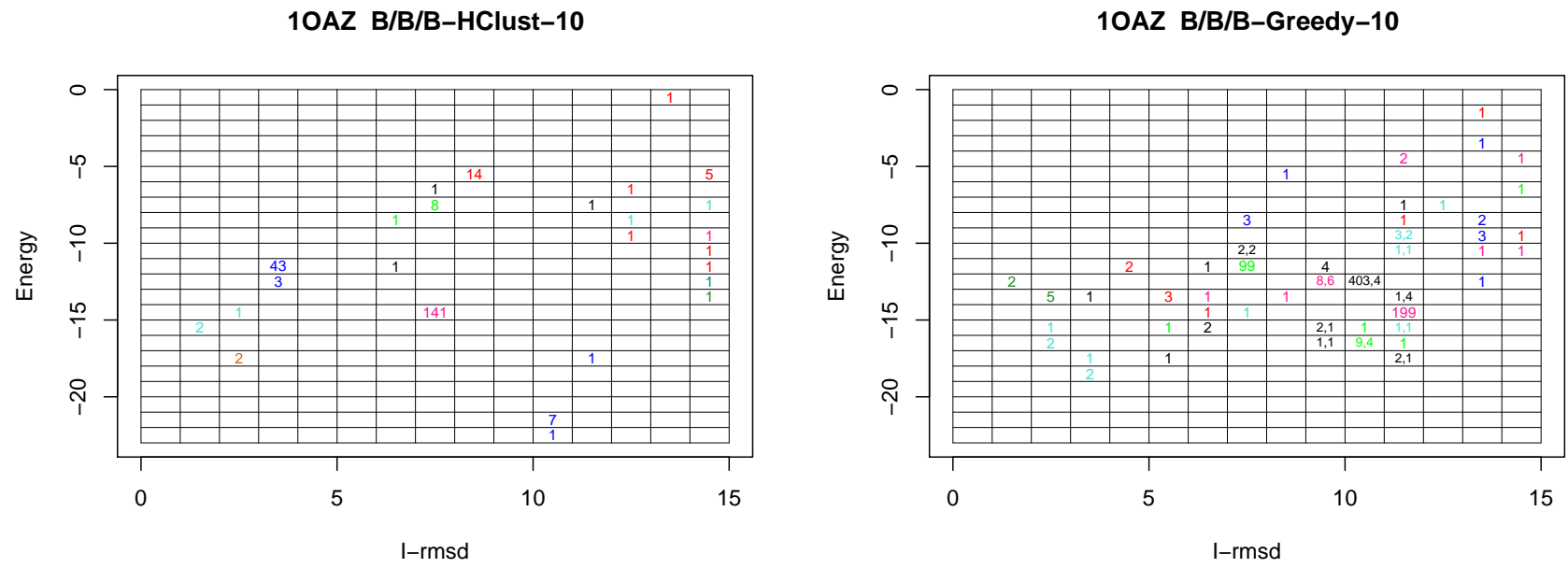

Fig. 19. 1OAZ: binning the docking tests using the Bound form of the receptor. See text for details.
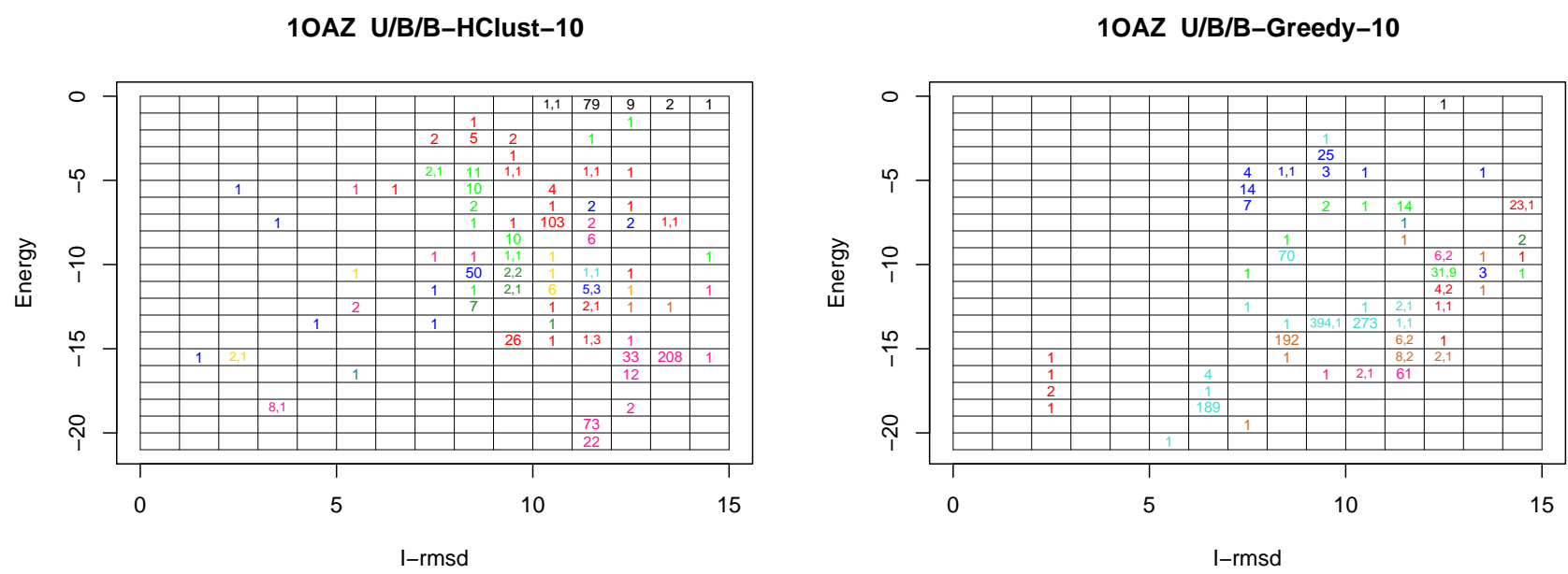

Fig. 20. 1OAZ: binning the docking tests using the Unbound form of the receptor. See text for details. 